\title{
MATHEMATICAL JUSTIFICATION OF A SHALLOW WATER MODEL*
}

\author{
DIDIER BRESCH ${ }^{\dagger}$ AND PASCAL NOBLE A $^{\ddagger}$
}

\begin{abstract}
The shallow water equations are widely used to model the flow of a thin layer of fluid submitted to gravity forces. They are usually formally derived from the full incompressible Navier-Stokes equations with free surface under the modeling hypothesis that the pressure is hydrostatic, the flow is laminar, gradually varied and the characteristic fluid height is small relative to the characteristics flow length. This paper deals with the mathematical justification of such asymptotic process assuming a non zero surface tension coefficient and some constraints on the data. we also discuss relation between lubrication models and shallow water systems with no surface tension coefficient necessity.
\end{abstract}

Key words. Navier-Stokes, shallow water, lubrication models, thin domain, free surface, asymptotic analysis, Sobolev spaces

AMS subject classifications. 35Q30, 35R35, 76A20, 76B45, 76D08

1. Introduction. The shallow water description through the Saint-Venant system is usual for many applications (rivers flow, tidal waves, oceans). Although SaintVenant derived it from elementary principle in 1871, one understands only from less than few years how to derive it formally from the Navier-Stokes equations with free surface and various bottom boundary conditions assuming the depth to be small enough, see for instance [16] and [8]-[11] for flat bottom. We also referred the reader to [4] and [5] for shallow water type equation with arbitrary topography. The flow these equations describe is the horizontal flow caused by changes in the height of the pressure surface of the fluid. Whereas the derivation of shallow water equations from the incompressible Navier-Stokes system with free surface, through a depth integration of continuity and momentum equations, is now understood, there is still a lack of mathematical justification of such approaches. In that paper, we present the derivation of a shallow water system, inspired from [16], that we justify rigorously. Note that the rigorous derivation is performed under further technical assumptions that are necessary to have smooth solutions.

First, after a suitable scaling of the Navier-Stokes equations with free surface boundary conditions and no slip condition at bottom, the solutions are formally expanded with respect to the aspect ratio $\varepsilon=H / L$ between the characteristic fluid height $H$ and the characteristic wavelength $L$ of flow variables. Substituting the asymptotic expansions into the depth averaged continuity and momentum equations, we obtain a shallow water system in a closed form plus a remainder that formally tends to 0 as $\varepsilon \rightarrow 0$.

To justify rigorously such asymptotic, see Theorem 1 and 2, we establish uniform estimates in classical Sobolev spaces on solutions of the full Navier-Stokes system with respect to $\varepsilon$ and we obtain that the remainder in the shallow water system tends to 0 as $\varepsilon \rightarrow 0$ as a consequence. Such shallow water approximate system reads, in a

\footnotetext{
*Received November 17, 2007; accepted for publication February 18, 2008.

†Université de Savoie, Laboratoire de Mathématiques, UMR CNRS 5127, Campus Scientifique, 73376 Le Bourget du Lac, France (Didier.Bresch@univ-savoie.fr).

${ }^{\ddagger}$ Université de Lyon, Université Lyon 1, UMR CNRS 5208, Institut Camille Jordan, Batiment du Doyen Jean Braconnier, 43, blvd du 11 novembre 1918, F - 69622 Villeurbanne Cedex, France (noble@math.univ-lyon1.fr).
} 
non-dimensional form,

$$
\left\{\begin{array}{l}
h_{t}+(h v)_{x}=0, \\
(h v)_{t}+\left(\frac{6}{5} h v^{2}+\frac{c h^{2}}{\mathbf{R e}}-\frac{(2 s)^{2}}{75} h^{5}\right)_{x} \\
\quad-\varepsilon^{2} W h h_{x x x}=\frac{1}{\varepsilon \mathbf{R e}}\left(2 s h-\frac{3 v}{h}\right)
\end{array}\right.
$$

where $h$ denotes the height of the free surface, $v$ is the vertical average of the horizontal velocity component. The other quantitites $c, s, W, \varepsilon, \mathbf{R e}$ are respectively $\cos \theta, \sin \theta$ where $\theta$ is the slope angle, the Weber coefficient, the aspect ratio of the domain and the Reynolds number given in (7).

Remark that, to the authors knowledge, such mathematical justification of shallow water system seems to be new. In our mathematical study Reynolds number is assumed to be fixed and some assumptions on various physical quantities sizes are done such as $\sin \theta / \sqrt{\varepsilon} \kappa$ and initial data small enough in some sense, see Theorem (1).

The paper is divided in three parts: The first section, inspired from the recent derivation in [16], concerns the formal derivation of a shallow water model. The second section deals with uniform estimates, with respect to the aspect ratio, of solutions to Navier-Stokes equations combining similar process than those used in [12], [14] and similar estimates than those in [13]. Using the previous sections, the third section concludes on the rigourous derivation of the shallow water model. We also give some comments around lubrication models which are included in the shallow water mathematical derivation with no surface tension coefficient necessity. The reader, interested by lubrication models, is referred for instance to [15], [9], [3].

2. Formal derivation of a shallow water model. In what follows, we derive formally a shallow water model from the incompressible Navier-Stokes equations with a free surface. The fluid flows downward to an inclined plane under the effect of gravity. The Navier-Stokes system comes with boundary conditions: we assume a no slip condition at the bottom and continuity of the fluid stress at the free surface, the fluid being submitted here to surface tension forces. Moreover, assuming that the layer of fluid is advected by the fluid velocity, we obtain an evolution equation for the fluid height. We first scale the equations in the shallow water setting. Then, we calculate formally an asymptotic axpansion of the flow variables with respect to the film parameter $\varepsilon$. Inserting the expansion in depth averaged continuity and momentum equation, we obtain a shallow water model and identify a remainder that formally tends to 0 as $\varepsilon \rightarrow 0$.

2.1. Scaling the incompressible Navier-Stokes system. In that paper, we consider a relatively thin layer of fluid flowing down an inclined plane, at an angle $\theta$ with respect to the horizontal, under the effect of gravity. Remark that we consider a flat bottom for simplicity. The reader is referred to [5] for formal derivation with arbitrary topography. The fluid is incompressible and the fluid density is constant and set to 1. The flow is supposed to be 2-dimensional. A coordinate system $(x, z)$ is defined as the $x$-axis downslope along and the $z$-axis upward normal to the plane bed. The longitudinal and transverse velocity are denoted $(u, w)$, the pressure by $p$ and the total flow depth by $h$. The fluid layer $\Omega_{t}$ is the set

$$
\Omega_{t}=\left\{(x, z) \in \mathbb{R}^{2}: \quad 0<z<h(t, x)\right\} .
$$


The incompressible Navier-Stokes equations read, for all $(x, z) \in \Omega_{t}$ :

$$
\left\{\begin{array}{l}
u_{t}+u u_{x}+w u_{z}+p_{x}=g \sin \theta+\nu \Delta u \\
w_{t}+u w_{x}+w w_{z}+p_{z}=-g \cos \theta+\nu \Delta w \\
u_{x}+w_{z}=0
\end{array}\right.
$$

The $\nu$ constant denotes the fluid viscosity and $g$ is the gravity constant. The NavierStokes equations is supplemented with boundary conditions. More precisely, we assume at the bottom a no-slip condition on a flat bottom:

$$
\left.u\right|_{z=0}=\left.w\right|_{z=0}=0 .
$$

At the free boundary, we assume that the atmospheric pressure $p_{\text {atm }}$ is constant and set to 0 . The fluid is submitted to surface tension forces. Hence, the continuity of the fluid stress at the free surface, written $\sigma n=\kappa n$ with $\sigma=-p \mathrm{Id}+\nu\left(\nabla u+\nabla^{t} u\right)$, yields the following conditions:

$$
\begin{aligned}
& \left.p\right|_{z=h}+\underline{\kappa} h_{x x}\left(1+h_{x}^{2}\right)^{-\frac{3}{2}}=-\left.2 \nu \frac{1+h_{x}^{2}}{1-h_{x}^{2}} u_{x}\right|_{z=h}, \\
& \left.u_{z}\right|_{z=h}+\left.w_{x}\right|_{z=h}=-\left.4 \frac{h_{x}}{1-h_{x}^{2}} w_{z}\right|_{z=h},
\end{aligned}
$$

where $\kappa$ measures the capillarity (the curvature). The layer fluid is advected by the speed $\vec{u}=(u, w)$, giving the evolution equation for the fluid height (the usual kinematic condition)

$$
h_{t}+\left.h_{x} u\right|_{z=h}=\left.w\right|_{z=h} .
$$

This set of equations possesses a classical steady solution, so called Nusselt flow. The height of the fluid $h(x, t)=H$ is constant, the transverse component velocity $w$ is equal to 0 and the longitudinal component speed $u$ has a parabolic dependence with respect to $z$

$$
U(x, z)=\frac{g}{2 \nu}\left(2 H z-z^{2}\right) \sin \theta .
$$

Moreover, the pressure is hydrostatic:

$$
p(x, z)=g(H-z) \cos \theta .
$$

Adimensionalization and rescaling. We shall use the Nusselt flow in the sequel to scale the Navier-Stokes equations. With the notation $U_{0}=g H^{2} / 2 \nu$, we define the rescaled variables

$$
\begin{array}{ll}
z=H \bar{z}, & h=H \bar{h}, \quad x=L \bar{x}, \quad \varepsilon=\frac{H}{L}, \\
u=U_{0} \bar{u}, & w=\varepsilon U_{0} \bar{w}, \quad p=g H \bar{p}, \\
t=\frac{L}{U_{0}} \bar{t}, & \mathbf{R e}=\frac{H U_{0}}{\nu}, \quad W=\frac{\kappa}{g H^{2}} .
\end{array}
$$

In what follows, we denote $c=\cos \theta, s=\sin \theta$. Under the shallow water scaling and dropping the ${ }^{-}$, the Navier-Stokes equations read for all $(x, z)$ in $\Omega_{t}$ :

$$
\begin{aligned}
u_{t}+u u_{x}+w u_{z}+\frac{2}{\mathbf{R e}} p_{x} & =\frac{2 s}{\varepsilon \mathbf{R e}}+\frac{1}{\varepsilon \mathbf{R e}}\left(\varepsilon^{2} u_{x x}+u_{z z}\right), \\
w_{t}+u w_{x}+w w_{z}+\frac{2}{\varepsilon^{2} \mathbf{R e}} p_{z} & =-\frac{2 c}{\varepsilon^{2} \mathbf{R e}}+\frac{1}{\varepsilon \mathbf{R e}}\left(\varepsilon^{2} w_{x x}+w_{z z}\right), \\
u_{x}+w_{z} & =0 .
\end{aligned}
$$


The no-slip condition $\left.u\right|_{z=0}=\left.w\right|_{z=0}=0$ and the evolution equation for $h$

$$
h_{t}+\left.h_{x} u\right|_{z=h}=\left.w\right|_{z=h},
$$

are unchanged whereas the continuity of the fluid stress reads

$$
\begin{aligned}
& \left.p\right|_{z=h}+\varepsilon^{2} W h_{x x}\left(1+\varepsilon^{2} h_{x}^{2}\right)^{-\frac{3}{2}}=-\left.\varepsilon \frac{1+\varepsilon^{2} h_{x}^{2}}{1-\varepsilon^{2} h_{x}^{2}} u_{x}\right|_{z=h}, \\
& \left.u_{z}\right|_{z=h}+\left.\varepsilon^{2} w_{x}\right|_{z=h}=-\left.\frac{4 \varepsilon^{2} h_{x}}{1-\varepsilon^{2} h_{x}^{2}} w_{z}\right|_{z=h} .
\end{aligned}
$$

We shall prove that in the asymptotic regime $\varepsilon \rightarrow 0$, the solutions of the NavierStokes equations (8) with rescaled boundary conditions are close to the stationnary Nusselt flow and have an asymptotic expansion with respect $\varepsilon$. This is done in the next section.

2.2. Asymptotic expansion of solutions. Under the shallow water scaling, the flow variables are close to a Nusselt flow. More precisely, we write the NavierStokes equations as a differential system in the crosstream variable $z$ with boundary conditions at $z=0$ and $z=h$. First, the longitudinal component velocity $u$ satisfies

$$
\begin{aligned}
& u_{z z}+2 s=2 \varepsilon p_{x}+\varepsilon \operatorname{Re}\left(u_{t}+u u_{x}+w u_{z}\right)-\varepsilon^{2} u_{x x} \\
& =\Psi_{u}(u, w, p)(t, x, z) \text {, } \\
& \left.u\right|_{z=0}=0, \\
& \left.u_{z}\right|_{z=h}=-\left.\varepsilon^{2} w_{x}\right|_{z=h}+\left.\frac{4 \varepsilon^{2}}{1-\varepsilon^{2} h_{x}^{2}} h_{x} u_{x}\right|_{z=h} \\
& =\Pi_{u}(u, w)(t, x) .
\end{aligned}
$$

The functions $\Psi_{u}(u, w, p), \Pi_{u}(u, w)$ satisfy formally the estimates

$$
\Psi_{u}(u, w, p)=\mathcal{O}(\varepsilon+\varepsilon \mathbf{R e}), \quad \Pi_{u}(u, w)=\mathcal{O}\left(\varepsilon^{2}\right) .
$$

Integrating (11) with respect to $z$, we obtain

$$
u(t, x, z)=2 s\left(h(t, x) z-\frac{z^{2}}{2}\right)+\mathcal{F}_{u}(u, w, p)(t, x, z),
$$

where the function $\mathcal{F}_{u}(u, w, p)$ is defined by

$$
\begin{aligned}
& \mathcal{F}_{u}(u, w, p)(t, x, z) \\
& \quad=z \Pi_{u}(u, w)(t, x)-\int_{0}^{z} \int_{\bar{z}}^{h} \Psi_{u}(u, w, p)(t, x, y) d y d \bar{z} .
\end{aligned}
$$

As a consequence, the longitudinal velocity component $u$ expands in the form

$$
u(t, x, z)=2 s\left(h(t, x) z-\frac{z^{2}}{2}\right)+\mathcal{O}(\varepsilon+\varepsilon \mathbf{R e}) .
$$

The profile for $u$ is then closed to a Nusselt type flow. We show in a similar way that the pressure is close to a hydrostatic distribution. More precisely, the pressure $p$ 
satisfies the "ordinary" differential equation with respect to $z$ :

$$
\begin{aligned}
p_{z}+c & =\frac{\varepsilon}{2}\left(w_{z z}+\varepsilon^{2} w_{x x}\right)-\frac{\varepsilon^{2} \mathbf{R e}}{2}\left(w_{t}+u w_{x}+w w_{z}\right) \\
& =\Psi_{p}(u, w)(t, x, z) \\
\left.p\right|_{z=h} & =-\bar{\kappa} \frac{\mathbf{R e}}{2} \frac{h_{x x}}{\left(1+\varepsilon^{2} h_{x}^{2}\right)^{\frac{3}{2}}}-\left.\varepsilon u_{x}\right|_{z=h} \frac{1+\varepsilon^{2} h_{x}^{2}}{1-\varepsilon^{2} h_{x}^{2}} \\
& =-\bar{\kappa} \frac{\mathbf{R e}}{2} h_{x x}+\Pi_{p}(u)(t, x),
\end{aligned}
$$

where the constant $\bar{\kappa}$, defined by $\bar{\kappa}=\varepsilon^{2} W$, is assumed to be of order $\mathcal{O}(1)$ in order to see the capillarity effects. The functions $\Psi_{p}(u, w), \Pi_{p}(u)$ satisfy the formal estimates

$$
\Psi_{p}(u, w)=\mathcal{O}(\varepsilon), \quad \Pi_{p}(u)=\mathcal{O}(\varepsilon) .
$$

We integrate (16) with respect to $z$ and find

$$
p(x, z, t)=-\bar{\kappa} \frac{\mathbf{R e}}{2} h_{x x}+c(h-z)+\mathcal{F}_{p}(u, w, p)(x, z, t),
$$

where the function $\mathcal{F}_{p}(u, w, p)$ is defined by

$$
\mathcal{F}_{p}(u, w, p)=\Pi_{p}(u)(x, t)-\int_{z}^{h} \Psi_{p}(u, w)(t, x, y) d y
$$

The pressure $p$ has, in a first approximation, an hydrostatic distribution and expands in the form

$$
p(x, z, t)=-\bar{\kappa} \frac{\mathbf{R e}}{2} h_{x x}+c(h-z)+\mathcal{O}(\varepsilon) .
$$

The transverse component velocity $w$ is determined, using the free divergence condition $w_{z}+u_{x}=0$ and the no-slip condition $w(x, 0)=0$. More precisely, we find $w(t, x, z)=-\int_{0}^{z} u_{x}(t, x, y) d y$. We deduce that $w$ has the asymptotic expansion

$$
w(t, x, z)=-\int_{0}^{z} u_{x}(t, x, y) d y=-s h_{x} z^{2}+\mathcal{O}(\varepsilon+\varepsilon \mathbf{R e}) .
$$

In the following, we describe an iterative scheme to compute a formal expansion of Navier-Stokes solutions to any order in $\varepsilon$. First, we define the functions $u^{(0)}$ and $p^{(0)}$ as

$$
\begin{aligned}
& u^{(0)}(t, x, z)=s\left(2 h(t, x) z-z^{2}\right), \\
& p^{(0)}(t, x, z)=-\bar{\kappa} \mathbf{R e} h_{x x} / 2+c(h(t, x)-z) .
\end{aligned}
$$

We write the equations $(13,18)$ in the form

$$
\left(\begin{array}{l}
u \\
p
\end{array}\right)=\left(\begin{array}{l}
u^{(0)} \\
p^{(0)}
\end{array}\right)+\mathcal{F}(u, p, \varepsilon),
$$

where the function $\mathcal{F}(u, p, \varepsilon)$ is defined by

$$
\mathcal{F}(u, p, \varepsilon)=\left(\begin{array}{c}
\mathcal{F}_{u}(u, w, p) \\
\mathcal{F}_{p}(u, w, p)
\end{array}\right), \quad w(t, x, z)=-\int_{0}^{z} u_{x}(t, x, y) d y .
$$


Then, any solution of the Navier-Stokes equations appears as a fixed point of an operator that is formally $\mathcal{O}(\varepsilon+\varepsilon \mathbf{R e})$-Lipschitz on any bounded set. Following the proof of the fixed point theorem, we define the sequence of functions $\left(u^{n}, w^{(n)}, p^{(n)}\right)_{n \geq 1}$ such that

$$
\left(\begin{array}{l}
u^{(n+1)} \\
p^{(n+1)}
\end{array}\right)=\left(\begin{array}{c}
u^{(0)} \\
p^{(0)}
\end{array}\right)+\mathcal{F}\left(u^{(n)}, p^{(n)}, \varepsilon\right),
$$

and $w^{(n)}(t, x, z)=-\int_{0}^{z} u_{x}^{(n)}(t, x, y) d y$.

We assume that all the derivatives of $(u, w, p)$ remain bounded and the sequence $\left(u^{n}, w^{(n)}, p^{(n)}\right)_{n \geq 1}$ is bounded. We can prove by induction the formal estimate

$$
\max \left(\left|u-u^{(n)}\right|,\left|w-w^{(n)}\right|,\left|p-p^{(n)}\right|\right)=\mathcal{O}\left((\varepsilon+\varepsilon \mathbf{R e})^{n+1}\right) .
$$

As a consequence, we clearly see that the $n$-th term of the sequence of functions $\left(u^{n}, w^{(n)}, p^{(n)}\right)_{n \geq 1}$ is a formal approximation of a solution of the full Navier-Stokes equations up to order $\mathcal{O}\left((\varepsilon+\varepsilon \mathbf{R e})^{n+1}\right)$. In what follows, we use that approximation of the solutions to close the depth average continuity and momentum equations and obtain a shallow water model.

2.3. The shallow water model. In the sequel, we write a shallow water model for the fluid height $h$ and the total discharge rate $h v=\int_{0}^{h} u(., y,) d$.$y . Here v$ represents the mean velocity along the depth flow. On the one hand, integrating the divergence free condition $u_{x}+w_{z}=0$ along the fluid height and using the kinematic equation for $h$ namely $h_{t}+\left.h_{x} u\right|_{z=h}=\left.w\right|_{z=h}$, we find

$$
h_{t}+\left(\int_{0}^{h} u(x, z) d z\right)_{x}=h_{t}+(h v)_{x}=0 .
$$

Note that the equation is exact and already in a closed form. Let us now write an evolution equation for $h v=\int_{0}^{h} u(\cdot, \cdot, \zeta) d \zeta$. For that purpose, we integrate the evolution equation on the velocity component $u$ along the flow depth and substitute the boundary conditions (10) into the resulting equation:

$$
\begin{gathered}
\left(\int_{0}^{h} u d z\right)_{t}+\left(\int_{0}^{h} u^{2} d z\right)_{x}+\frac{2}{\mathbf{R e}}\left(\int_{0}^{h} p d z\right)_{x}+\bar{\kappa} \frac{h_{x x}}{\left(1+\varepsilon^{2} h_{x}^{2}\right)^{\frac{3}{2}}} h_{x}= \\
\frac{1}{\varepsilon \mathbf{R e}}\left(2 s h-u_{z}(x, 0)\right)+\frac{\varepsilon}{\operatorname{Re}}\left(\int_{0}^{h} 2 u_{x} d z\right)_{x} .
\end{gathered}
$$

In order to write a momentum equation in a closed form, we shall calculate an expansion of the averaged quantities in (28) with respect to $\varepsilon, h, v$. First, let us note that

$$
\begin{aligned}
(h v)(t, x) & =\int_{0}^{h(t, x)} u(t, x, \zeta) d \zeta=\int_{0}^{h(t, x)} u^{(0)}(t, x, \zeta) d \zeta+\int_{0}^{h(t, x)} \delta u(t, x, \zeta) d \zeta, \\
& =2 s \frac{h^{3}}{3}+\int_{0}^{h(t, x)} \delta u(t, x, \zeta) d \zeta .
\end{aligned}
$$


Here, the function $\delta u$ is simply $\delta u=u-u^{(0)}$. From the previous section, we deduce the formal estimate $\delta u=\mathcal{O}(\varepsilon+\varepsilon \mathbf{R e})$. We compute the average quantities $\int_{0}^{h} u^{2}$ and $\int_{0}^{h} p$ :

$$
\begin{aligned}
\int_{0}^{h(t, x)} u^{2}(t, x, \zeta) d \zeta & =\int_{0}^{h(t, x)}\left(u^{(0)}\right)^{2}(t, x, \zeta) d \zeta+\int_{0}^{h(t, x)}\left(u^{2}-\left(u^{(0)}\right)^{2}\right)(t, x, \zeta) d \zeta \\
& =\frac{8 h(t, x)}{15}\left(s h^{2}(t, x)\right)^{2}+\int_{0}^{h(t, x)}\left(u^{2}-\left(u^{(0)}\right)^{2}\right)(t, x, \zeta) d \zeta
\end{aligned}
$$

We substitute $2 s h^{2}=3 v-\frac{1}{h} \int_{0}^{h} \delta u$ into (29). The average quantity $\int_{0}^{h} u^{2}$ reads

$$
\int_{0}^{h(t, x)} u^{2}(t, x, \zeta) d \zeta=\frac{6}{5}\left(h v^{2}\right)(t, x)+\mathcal{R}_{1}^{(1)}
$$

where the function $\mathcal{R}_{1}^{(1)}$ is defined by

$$
\begin{aligned}
\mathcal{R}_{1}^{(1)}(t, x)= & \int_{0}^{h(t, x)}\left(u^{2}-\left(u^{(0)}\right)^{2}\right)(t, x, \zeta) d \zeta \\
& +\frac{12}{15} v \int_{0}^{h(t, x)} \delta u(t, x, \zeta) d \zeta+\frac{2}{15 h(t, x)}\left(\int_{0}^{h(t, x)} \delta u(t, x, \zeta) d \zeta\right)^{2} .
\end{aligned}
$$

Inserting the estimate $u-u^{(0)}=\mathcal{O}(\varepsilon+\varepsilon \mathbf{R e})$ into the expression for $\mathcal{R}_{1}$, we find that, formally, $\mathcal{R}_{1}=\mathcal{O}(\varepsilon+\varepsilon \mathbf{R e})$. We easily compute the average quantity $\int_{0}^{h} p$ :

$$
\int_{0}^{h} p(\cdot, \cdot, \zeta) d \zeta=-\bar{\kappa} \frac{\mathbf{R e}}{2} h h_{x x}+c \frac{h^{2}}{2}+\mathcal{R}_{1}^{(2)}
$$

with

$$
\mathcal{R}_{1}^{(2)}(t, x)=\int_{0}^{h(t, x)}\left(p-p^{(0)}\right)(t, x, \zeta) d \zeta
$$

We easily prove that, formally, $\mathcal{R}_{1}^{(2)}$ satisfies the estimates $\mathcal{R}_{1}^{(2)}=\mathcal{O}(\varepsilon+\varepsilon \mathbf{R e})$. Substituting (30),(31) into (28), one obtains

$$
(h v)_{t}+\left(\frac{6}{5} h v^{2}+\frac{2}{\mathbf{R e}} c \frac{h^{2}}{2}\right)_{x}-\bar{\kappa} h h_{x x x}=\frac{1}{\varepsilon \mathbf{R e}}\left(2 s h-u_{z}(x, 0)\right)+\mathcal{R}_{1},
$$

where the function $\mathcal{R}_{1}$ is defined by

$$
\begin{aligned}
\mathcal{R}_{1}= & -\partial_{x} \mathcal{R}_{1}^{(1)}-\partial_{x} \mathcal{R}_{1}^{(2)} \\
& +\frac{\varepsilon}{\operatorname{Re}} \partial_{x}\left(\int_{0}^{h} u_{x}(\cdot, \cdot, \zeta) d \zeta\right)+\bar{\kappa}\left(\left(1-\left(1+\varepsilon^{2} h_{x}^{2}\right)^{-\frac{3}{2}}\right) h_{x} h_{x x}\right)
\end{aligned}
$$

and satisfies the formal estimate

$$
\mathcal{R}_{1}=\mathcal{O}\left(\varepsilon\left(R e+1+R e^{-1}\right)\right) .
$$


Dropping the "small" term $\mathcal{R}_{1}$, we can see that the momentum equation (32) is almost in a closed form and yields a shallow water model for $h, h v$. There remains to eliminate $u_{z}(x, 0)$. Due to the presence of the singular factor $1 /(\varepsilon \mathbf{R e})$, we cannot directly use the expansion

$$
u_{z}(0)=u_{z}^{(0)}(0)+\mathcal{O}(\varepsilon+\varepsilon \mathbf{R e})=\frac{3 v}{h}+\mathcal{O}(\varepsilon+\varepsilon \mathbf{R e}) .
$$

In that case, the remainder would be of order $\mathcal{O}(1)$. As a consequence, we need an asymptotic expansion of $u_{z}(0)$ and $h v$ with respect to $\varepsilon$ and function of $h$ and its derivatives up to order 1 . For that purpose, we use the function $u^{(1)}$ introduced in the previous section. Recall that we have the estimate $u-u^{(1)}=\mathcal{O}\left((\varepsilon+\varepsilon \mathbf{R e})^{2}\right)$. We aim to compute an expansion of $h v$ and $u_{z}(., 0)$ up to order 1: let us expand $u$ with respect to $\varepsilon$. We find, using the iterative scheme introduced previously that

$$
u=u^{(0)}-\int_{0}^{z} \int_{y}^{h} 2 \varepsilon p^{(0)}+\varepsilon \mathbf{R e}\left(u_{t}^{(0)}+u^{(0)} u_{x}^{(0)}+w^{(0)} u_{z}^{(0)}\right)+\varepsilon \mathbf{R e} \mathcal{R}_{2}^{(1)},
$$

where the notation $\int_{0}^{z} \int_{y}^{h} f$ is used to defined the function of $(t, x, z)$ as

$$
\int_{0}^{z} \int_{y}^{h} f(t, x)=\int_{0}^{z} \int_{y}^{h(t, x)} f(t, x, \bar{z}) d \bar{z} d y .
$$

Moreover the function $\mathcal{R}_{2}^{(1)}$ is defined as

$$
\begin{aligned}
\mathcal{R}_{2}^{(1)}= & \frac{\varepsilon}{\mathbf{R e}} \int_{0}^{z} \int_{y}^{h} u_{x x}-u_{x x}^{(0)}-\int_{0}^{z} \int_{y}^{h} \frac{2}{\mathbf{R e}}\left(p-p^{(0)}\right)_{x} \\
& -\frac{\varepsilon z}{\mathbf{R e}}\left(\left(w_{x}(h)+\frac{w_{z}(h)}{1-\varepsilon^{2} h_{x}^{2}}\right)-\left(w_{x}^{(0)}(h)+\frac{w_{z}^{(0)}(h)}{1-\varepsilon^{2} h_{x}^{2}}\right)\right) \\
& +\int_{0}^{z} \int_{y}^{h}\left(u-u^{(0)}\right)_{t}+\left(u u_{x}-u^{(0)} u_{x}^{(0)}\right)+\left(w u_{z}-w^{(0)} u_{z}^{(0)}\right) .
\end{aligned}
$$

It is easily seen that $\mathcal{R}_{2}^{(1)}=\mathcal{O}\left(\varepsilon\left(1+\mathbf{R e}+\mathbf{R} \mathbf{e}^{-1}\right)\right)$. In order to simplify the notations, we denote $\bar{u}^{(1)}$ the function

$$
\bar{u}^{(1)}=-2 p^{(0)}-\boldsymbol{\operatorname { R e }}\left(u_{t}^{(0)}+u^{(0)} u_{x}^{(0)}+w^{(0)} u_{z}^{(0)}\right) .
$$

Then, the component velocity $u$ expands in the form

$$
u=u^{(0)}+\varepsilon \bar{u}^{(1)}+\varepsilon \mathbf{R e} \mathcal{R}_{2}^{(1)} .
$$

From (35), we deduce that

$$
\begin{aligned}
& \left.u_{z}\right|_{z=0}=2 s h+\left.\varepsilon \partial_{z} \bar{u}^{(1)}\right|_{z=0}+\left.\varepsilon \mathbf{R e} \partial_{z} \mathcal{R}_{2}^{(1)}\right|_{z=0}, \\
& (h v)(t, x)=2 s \frac{h^{3}(t, x)}{3}+\varepsilon \int_{0}^{h(t, x)} \bar{u}^{(1)}(t, x, \zeta) d \zeta+\varepsilon \mathbf{R e} \int_{0}^{h(t, x)} \mathcal{R}_{2}^{(1)}(t, x, \zeta) d \zeta .
\end{aligned}
$$


Then, eliminating $2 s h$ from the expansion of $\left.u_{z}\right|_{z=0}$, one obtains the asymptotic expansion

$$
\left.u_{z}\right|_{z=0}=\frac{3 v}{h}+\varepsilon\left(\left.\partial_{z} \bar{u}^{(1)}\right|_{z=0}-\frac{3}{h^{2}} \int_{0}^{h} \bar{u}^{(1)}\right)+\varepsilon \mathbf{R e}\left(\left.\partial_{z} \mathcal{R}_{2}^{(1)}\right|_{z=0}-\frac{3}{h^{2}} \int_{0}^{h} \mathcal{R}_{2}^{(1)}\right) .
$$

We substitute the expansion (36) into the momentum equation (32)

$$
(h v)_{t}+\left(\frac{6}{5} h v^{2}+\frac{2}{\mathbf{R e}} c \frac{h^{2}}{2}\right)_{x}-\bar{\kappa} h h_{x x x}=\frac{1}{\varepsilon \mathbf{R e}}\left(2 s h-\frac{3 v}{h}\right)-\tau+\mathcal{R}_{1}+\mathcal{R}_{2},
$$

with $\tau=\frac{7}{120}(2 s)^{2} h^{4} h_{x}+\frac{1}{8}(2 s) h^{2} h_{t}$ and $\mathcal{R}_{2}=\left.\partial_{z} \mathcal{R}_{2}^{(1)}\right|_{z=0}-\frac{3}{h^{2}} \int_{0}^{h} \mathcal{R}_{2}^{(1)}$.

Dropping the "small" term $\mathcal{R}=\mathcal{R}_{1}+\mathcal{R}_{2}=\mathcal{O}\left(\varepsilon\left(1+\mathbf{R e}+\mathbf{R} \mathbf{e}^{-1}\right)\right)$, we obtain a shallow water model in a closed form

$$
\left\{\begin{array}{l}
h_{t}+(h v)_{x}=0 \\
(h v)_{t}+\left(\frac{6}{5} h v^{2}+\frac{2}{\mathbf{R e}} c \frac{h^{2}}{2}\right)_{x}-\bar{\kappa} h h_{x x x}=\frac{1}{\varepsilon \mathbf{R e}}\left(2 s h-\frac{3 v}{h}\right)-\tau
\end{array}\right.
$$

The term $\tau$ can be written as the sum of a conservative term and a remainder of order $\mathcal{O}(\varepsilon+\varepsilon \mathbf{R e})$. More precisely, we substitute the expansion

$$
h v=2 s \frac{h^{3}}{3}+\int_{0}^{h}\left(u-u^{(0)}\right),
$$

into the conservation law $h_{t}+(h v)_{x}=0$. As a result we find that

$$
h_{t}=-2 s h^{2} h_{x}-\partial_{x}\left(\int_{0}^{h}\left(u-u^{(0)}\right)\right) \text {, }
$$

and the function $\tau$ reads $\tau=-\frac{(2 s)^{2}}{75}\left(h^{5}\right)_{x}-\frac{s h^{2}}{4} \partial_{x}\left(\int_{0}^{h}\left(u-u^{(0)}\right)\right)$. If we introduce the notation $\widetilde{\mathcal{R}}=\mathcal{R}+\frac{s h^{2}}{4} \partial_{x}\left(\int_{0}^{h} u-u^{(0)}\right)$, the momentum equation (37) reads

$$
(h v)_{t}+\left(\frac{6}{5} h v^{2}+\frac{2}{\mathbf{R e}} c \frac{h^{2}}{2}-\frac{(2 s)^{2}}{75} h^{5}\right)_{x}-\bar{\kappa} h h_{x x x}=\frac{1}{\varepsilon \mathbf{R e}}\left(2 s h-\frac{3 v}{h}\right)+\widetilde{\mathcal{R}},
$$

The remainder $\widetilde{\mathcal{R}}$ is formally of order $\mathcal{O}\left(\varepsilon\left(1+\mathbf{R} \mathbf{e}+\mathbf{R e}^{-1}\right)\right)$. Dropping this "small" term, we obtain a conservative form of the shallow water model (38):

$$
\left\{\begin{array}{l}
h_{t}+(h v)_{x}=0, \\
(h v)_{t}+\left(\frac{6}{5} h v^{2}+\frac{c h^{2}}{\mathbf{R e}}-\frac{(2 s)^{2}}{75} h^{5}\right)_{x} \\
-\bar{\kappa} h h_{x x x}=\frac{1}{\varepsilon \mathbf{R e}}\left(2 s h-\frac{3 v}{h}\right) .
\end{array}\right.
$$

This concludes the formal derivation of a shallow water model from the full NavierStokes equations with free surface. Formally, the remainder term $\mathcal{R}$ (resp. $\widetilde{\mathcal{R}}$ ) tends to 0 as $\varepsilon \rightarrow 0$. In what follows, we compute a priori estimates in classical Sobolev norms on the solutions $(u, w, p)$ of the full Navier-Stokes system. We shall prove rigorously that the convergence of $\mathcal{R}$ (resp. $\widetilde{\mathcal{R}}$ ) to 0 holds true in a suitable norm and thus justify rigorously the formal derivation presented here. Finally, we can easily obtain a shallow water model without capillarity, setting the capillary coefficient $\bar{\kappa}$ to 0 in (40). However, we shall see later on that capillarity is important to obtain energy estimates on solutions to Navier-Stokes equations and to justify the asymptotic process. 
3. Uniform estimates and Navier-Stokes Eqs. In that section, we compute a priori estimates of solutions of the full Navier-Stokes equations in the shallow water scaling. The method is based on the energy estimates obtained by T. Nishida et al. [12] to prove the existence of global solutions to the Navier-Stokes flows down an inclined plane. First, the fluid domain is transformed into a fixed domain through a suitable change of variable. Then, the Navier-Stokes equations are written in the neighbourhood of a Nusselt flow and the authors derive a priori estimates on the perturbations of that steady solutions. Classically, for sufficiently "small" initial data, the solutions are proved to be global solutions and decays to 0 as $t \rightarrow \infty$. In order to simplify the discussion, the functions are periodic in the $x$-direction. This is the point of view adopted in that paper. The main issue here is to obtain estimates in the shallow water asymptotic regime $\varepsilon \rightarrow 0$. The shallow water scaling make the diffusion term anisotropic: in order to adapt the method of T. NISHIDA et al., we have chosen to transform the fixed domain into a thin domain of size $\varepsilon$ in the $z$-direction. In that setting, the diffusion is isotropic and we can completely follows the approach of $\mathrm{T}$. Nishida et al.. Their estimates are mainly based on estimates on the Stokes problem. At this stage, we follow ideas of R. Temam and M. Ziane [13] on the existence of solutions to Navier-Stokes equations in thin and fixed domain: in particular they obtain Sobolev inequalities and estimates on the Stokes problem in a thin domain. As a result of this analysis, provided that the perturbation of the Nusselt flow is small in a suitable weighted norm, we prove uniform estimates on solutions of the NavierStokes equations in the shallow water regime $\varepsilon \rightarrow 0$. In all the paper long, we will denote $\|\cdot\|_{r}$ and $|\cdot|_{r}$ the $L^{r}$ norm respectively in two-dimensional in space domains and in the one-dimensional torus $\mathbb{T}$.

3.1. Preliminaries. In that section, we follow the approach initiated by J.T. BEALE $[1,2]$ to prove the existence of solutions to the Navier-Stokes equations with a free boundary. We assume that the functions are periodic in the $x$ variable and consider small perturbations of the Nusselt flow: for a suitable change of variable, we write the Navier-Stokes equations in a fixed thin domain. Then, we recall briefly the fundamental inequalities in thin domains that are used in the paper: Sobolev inequalities and estimates for the Stokes problem in thin domains.

3.1.1. Reduction of N-S equations to a fixed thin domain. The rescaled Navier-Stokes equations are given in $\Omega_{t}$ by

$$
\begin{aligned}
u_{t}+u u_{x}+w u_{z}+\frac{2}{\mathbf{R e}} p_{x} & =\frac{2 s}{\varepsilon \mathbf{R e}}+\frac{1}{\varepsilon \mathbf{R e}}\left(\varepsilon^{2} u_{x x}+u_{z z}\right), \\
w_{t}+u w_{x}+w w_{z}+\frac{2}{\varepsilon^{2} \mathbf{R e}} p_{z} & =-\frac{2 c}{\varepsilon^{2} \mathbf{R e}}+\frac{1}{\varepsilon \mathbf{R e}}\left(\varepsilon^{2} w_{x x}+w_{z z}\right), \\
u_{x}+w_{z} & =0
\end{aligned}
$$

where $\Omega_{t}=\{(x, z) \in \mathbb{T} \times \mathbb{R}: 0<z<h(t, x)\}$ is the fluid domain. The system is supplemented with boundary conditions: The no-slip condition on the bottom

$$
\left.u\right|_{z=0}=\left.w\right|_{z=0}=0,
$$

and the kinematic equation at surface for $h$

$$
h_{t}+\left.h_{x} u\right|_{z=h}=\left.w\right|_{z=h} .
$$


The continuity of the fluid stress reads

$$
\begin{aligned}
& \left.p\right|_{z=h}+\varepsilon^{2} W h_{x x}\left(1+\varepsilon^{2} h_{x}^{2}\right)^{-\frac{3}{2}}=-\left.\varepsilon u_{x}\right|_{z=h} \frac{1+\varepsilon^{2} h_{x}^{2}}{1-\varepsilon^{2} h_{x}^{2}}, \\
& \left.u_{z}\right|_{z=h}+\left.\varepsilon^{2} w_{x}\right|_{z=h}=-\left.\frac{4 \varepsilon^{2} h_{x}}{1-\varepsilon^{2} h_{x}^{2}} w_{z}\right|_{z=h} .
\end{aligned}
$$

In order to simplify the N-S equations, we introduce the hydrostatic correction of the pressure $\tilde{p}=p-c z$. We also assume that $h=1+\tilde{h}$ and $\tilde{h}$ is small. We clearly see that, in the shallow water scaling, the diffusion is anisotropic: in order to recover an isotropic viscosity and applies the idea of T. Nishida et al. [12], we write the equations in a thin domain and introduce the functions $\tilde{u}, \tilde{w}$ and $P$ so that

$$
u(t, x, z)=\tilde{u}(t, x, \varepsilon z), \quad \varepsilon w(t, x, z)=\tilde{w}(t, x, \varepsilon z), \quad P=\frac{\bar{p}}{\varepsilon} .
$$

We drop the $\approx$ for the sake of simplifying the notations. The divergence free equation reads $u_{x}+w_{z}=0$, whereas the momentum equations are given, for all $(x, z) \in$ $\mathbb{T} \times(0, \varepsilon(1+h))$, by

$$
\begin{aligned}
& u_{t}+u u_{x}+w u_{z}+2 \mu P_{x}=\frac{2 \mu}{\varepsilon^{2}} s+\mu \Delta u, \\
& w_{t}+u w_{x}+w w_{z}+2 \mu P_{z}=\mu \Delta w,
\end{aligned}
$$

where $\mu$ is the rescaled viscosity $\mu=\frac{\varepsilon}{\mathbf{R e}}$. In that setting, we write the continuity of the fluid stress at the boundary $z=\varepsilon(1+h)$ in the form

$$
\begin{aligned}
& P-\frac{c}{\varepsilon}(1+h)+\varepsilon W h_{x x}\left(1+\varepsilon^{2} h_{x}^{2}\right)^{-\frac{3}{2}}=-u_{x} \frac{1+\varepsilon^{2} h_{x}^{2}}{1-\varepsilon^{2} h_{x}^{2}}, \\
& u_{z}+w_{x}=-\frac{4 \varepsilon h_{x}}{1-\varepsilon^{2} h_{x}^{2}} w_{z} .
\end{aligned}
$$

The no-slip condition is unchanged and the kinematic equation for $h$ reads in $\mathbb{T}$

$$
h_{t}+\left.h_{x} u\right|_{z=\varepsilon(1+h)}=\frac{\left.w\right|_{z=\varepsilon(1+h)}}{\varepsilon} .
$$

In that framework, the steady solution, so called Nusselt flow, is defined by $h=0$ and

$$
U(x, z)=\frac{s}{\varepsilon^{2}}\left(2 \varepsilon z-z^{2}\right), \quad P=\frac{c}{\varepsilon} .
$$

We consider the Navier-Stokes equations in the neighbourhood of the Nusselt flow and introduce the functions $\tilde{u}, \tilde{P}$ so that

$$
u=U+\tilde{u}, \quad P=\frac{c}{\varepsilon}+\tilde{P} .
$$

We remove the $\tilde{r}$ to simplify the notation. The momentum equations read:

$$
\begin{aligned}
u_{t}+(U+u) u_{x}+w\left(U_{z}+u_{z}\right)+2 \mu P_{x} & =\mu \Delta u, \\
w_{t}+(U+u) w_{x}+w w_{z}+2 \mu P_{z} & =\mu \Delta w .
\end{aligned}
$$

The divergence free equation is written

$$
u_{x}+w_{z}=0
$$


The boundary conditions at the free surface $z=\varepsilon(1+h)$ are given by

$$
\begin{aligned}
& P-\frac{c}{\varepsilon} h+\varepsilon W h_{x x}\left(1+\varepsilon^{2} h_{x}^{2}\right)^{-\frac{3}{2}}=-u_{x} \frac{1+\varepsilon^{2} h_{x}^{2}}{1-\varepsilon^{2} h_{x}^{2}}, \\
& u_{z}+w_{x}-\frac{2 s}{\varepsilon} h=-\frac{4 \varepsilon h_{x}}{1-\varepsilon^{2} h_{x}^{2}} w_{z} .
\end{aligned}
$$

We obtain for the evolution equation for $h$

$$
h_{t}+\left(s\left(1-h^{2}\right)+u(., \varepsilon(1+h))\right) h_{x}=\frac{w(., \varepsilon(1+h))}{\varepsilon} .
$$

In the sequel, following the approach initiated by J.T. BEALE $[1,2]$, we aim to write the N-S equations (49),(50), in the fixed thin domain $\Omega=\mathbb{T} \times(0, \varepsilon)$. For that purpose, let us first remark that, integrating the divergence free condition along the flow depth and using the boundary condition (52), one finds

$$
h_{t}+\partial_{x}\left(\int_{0}^{(\varepsilon(1+h))} u\right)=0 .
$$

As a consequence, we obtain $\frac{d}{d t} \int_{\mathbb{T}} h(x, t) d x=0$ and the spatial mean of $h$ is constant. In the sequel, we assume $\langle h\rangle=\int_{\mathbb{T}} h(x, t) d x=0$. Under that hypothesis, we define $\bar{h}$ the extension of $h$ to $\Omega$ as:

$$
\bar{h}(t, x, z)=\sum_{k \neq 0} \frac{h_{k}(t)}{1+k^{2}(z-\varepsilon)^{2}} e^{i k x}, \forall x \in \mathbb{T} .
$$

Now, we define the change of variable from $\Omega$ to $\Omega_{t}$ in the form:

$$
\begin{gathered}
\Theta_{t}: \mathbb{T} \times(0, \varepsilon) \rightarrow \mathbb{T} \times(0, \varepsilon(1+\bar{h})), \\
(x, z) \mapsto(x, z(1+\bar{h}(t, x, z))) .
\end{gathered}
$$

The Jacobian matrix of the transformation is

$$
D \Theta_{t}=\left(\begin{array}{cc}
\frac{1}{z \bar{h}_{x}} & 1+\partial_{z}(z \bar{h})
\end{array}\right)
$$

The function $\Theta_{t}$ is then a diffeomorphism between $\Omega$ and $\Omega_{t}$ provided that $J=$ $1+\partial_{z}(z \bar{h})>0$ ( $(\bar{h}$ is supposed to be small). In order to preserve the divergence free condition, we introduce the Jacobian decomposition of the fluid velocity

$$
\left(\begin{array}{c}
u \\
w
\end{array}\right)=\frac{1}{J} D \Theta_{t} \circ \Theta_{t}^{-1}\left(\begin{array}{c}
\tilde{u} \\
\tilde{w}
\end{array}\right) \circ \Theta_{t}^{-1} .
$$

Dropping the $\sim$ from the equations, the momentum equations transform into

$$
\begin{aligned}
& u_{t}+U u_{x}+U_{z} w+2 \mu p_{x}=\mu \Delta u+F_{1}, \\
& w_{t}+U w_{x}+2 \mu p_{z}=\mu \Delta w+F_{2},
\end{aligned}
$$


whereas the divergence free condition $u_{x}+w_{z}=0$ is preserved. The functions $F_{i}, i=$ 1,2 in (57) contain the nonlinear terms:

$$
\begin{aligned}
F_{1} & =\frac{J_{t} u}{J}+h_{t} z \partial_{z}\left(\frac{u}{J}\right)-\frac{u}{J}\left(u_{x}-J_{x} u-h_{x} z\left(\frac{u_{z}}{J}\right)-\frac{J_{z} u}{J^{2}}\right) \\
& +U\left(J_{x} u+z h_{x} \partial_{z}\left(\frac{u}{J}\right)\right)-z h_{x} u\left(U_{z}+\frac{1}{J} \partial_{z}\left(\frac{u}{J}\right)\right)-w \partial_{z}\left(\frac{u}{J}\right) \\
& +2 \mu\left(h_{x} z p_{z}+(1-J) p_{x}\right)+\mu\left(2 J u_{x} \partial_{x}\left(\frac{1}{J}\right)+J u \partial_{x x}\left(\frac{1}{J}\right)\right. \\
& -J \partial_{x}\left(\frac{h_{x} z}{J} \partial_{z}\left(\frac{u}{J}\right)\right)-h_{x} z \partial_{x z}\left(\frac{u}{J}\right)+h_{x} z \partial_{z}\left(\frac{h_{x} z}{J} \partial_{z}\left(\frac{u}{J}\right)\right) \\
& \left.-\partial_{z}\left(\frac{1}{J}\right) \partial_{z}\left(\frac{u}{J}\right)+\left(J^{-2}-1\right) \partial_{z z} u+\frac{2}{J} \partial_{z}\left(\frac{1}{J}\right) u_{z}+\frac{u}{J} \partial_{z z}\left(\frac{1}{J}\right)\right), \\
F_{2} & =-\partial_{x}\left(\frac{h_{t} z u}{J}\right)+\frac{h_{x} z}{J} U u_{x}+\frac{h_{x} z}{J} U_{z} w-\frac{J_{x}}{J^{2}} u w-\frac{h_{x x} z}{J} u^{2} \\
& +\frac{u_{x} w}{J}+\left(\frac{h_{x} z}{J}\right)^{2} U_{z} u-\frac{u w_{x}}{J}-\frac{h_{x x} z}{J} U u-\frac{\left(h_{x} z\right)^{2}}{J} U \partial_{z}\left(\frac{u}{J}\right) \\
& +2 \mu\left(\frac{\partial_{z}\left(h_{x} z\right)-\left(h_{x} z\right)^{2}}{J} \partial_{z} p+h_{x} z p_{x}\right)+\mu\left(\left(1-J^{-2}\right) u_{z x}\right. \\
& \left.+2 \frac{h_{x} z}{J} u_{x x}-\left(\frac{h_{x} z}{J}\right)^{2} u_{z x}\right)+\mu\left(-2 \partial_{x}\left(\frac{h_{x} z J_{x} u}{J^{2}}\right)\right. \\
& +\partial_{x}\left(\frac{h_{x} z}{J}\right) \frac{J_{x} u}{J}-\partial_{x}\left(h_{x} z\right) \partial_{z}\left(\frac{u}{J}\right)+2 \partial_{x}\left(h_{x} z\right) \partial_{x}\left(\frac{u}{J}\right)+\partial_{x x}\left(h_{x} z\right) \frac{u}{J} \\
& +\partial_{x}\left(\frac{h_{x} z}{J} u\right)-\frac{h_{x} z}{J} \partial_{z}\left(\frac{h_{x} z}{J}\right) u_{x}+\frac{h_{x} z}{J}\left(\frac{J_{x}}{J}-\frac{h_{x} z J_{z}}{J^{2}}\right) \frac{J_{x} u}{J} \\
& \left.+2\left(\frac{h_{x} z}{J}\right)^{2} J_{x} \partial_{z}\left(\frac{u}{J}\right)+\left(\frac{h_{x} z}{J}\right)^{2} \frac{J_{x z} u}{J}+\frac{J_{x z} u}{J^{2}}+\frac{2 J_{x}}{J^{2}} \partial_{z}\left(\frac{u}{J}\right)-\frac{J_{x} J_{z} u}{J^{4}}\right) .
\end{aligned}
$$

In that setting, the continuity of the fluid stress at the fixed boundary $z=\varepsilon$ reads, for all $x$ in $\mathbb{T}$ :

$$
\begin{aligned}
& \left.u_{z}\right|_{z=\varepsilon}+\left.w_{x}\right|_{z=\varepsilon}-2 \frac{s}{\varepsilon} h=H_{1}, \\
& \left.P\right|_{z=\varepsilon}-\frac{c}{\varepsilon} h+\varepsilon W h_{x x}+\left.u_{x}\right|_{z=\varepsilon}=H_{2},
\end{aligned}
$$

where the functions $H_{i}, i=1,2$ contain the nonlinear terms and are given by

$$
\begin{aligned}
H_{1}= & \frac{4 \varepsilon}{1-\varepsilon^{2} h_{x}^{2}}\left(\frac{u_{x}}{1+h}-\frac{h_{x}}{(1+h)^{2}} \partial_{z}(z u)+\varepsilon^{2} \frac{h_{x} h_{z z} u}{(1+h)^{2}}\right) \\
& -\varepsilon \partial_{x}\left(\frac{h_{x} u}{1+h}\right)+\frac{\varepsilon h_{x}}{1+h}\left(w_{z}+\frac{\varepsilon h_{x} u_{z}}{1+h}+u \partial_{z}\left(\frac{h_{x} z}{J}\right)\right)+\varepsilon \frac{h_{z z} u}{(1+h)^{3}}, \\
H_{2}= & \varepsilon W h_{x x}\left(1-\left(1+\varepsilon^{2} h_{x}^{2}\right)^{-\frac{3}{2}}\right)+\frac{h u_{x}}{1+h}+\frac{u_{x}}{1+h}\left(1-\frac{1+\varepsilon^{2} h_{x}^{2}}{1-\varepsilon^{2} h_{x}^{2}}\right) \\
& +\frac{1+\varepsilon^{2} h_{x}^{2}}{1-\varepsilon^{2} h_{x}^{2}}\left(\frac{h_{x}}{(1+h)^{2}} \partial_{z}(z u)-\varepsilon^{2} \frac{h_{x} h_{z z} u}{(1+h)^{3}}\right) .
\end{aligned}
$$

Finally, the time evolution for $h$ is described by the equation in $\mathbb{T}$.

$$
h_{t}+s\left(1-h^{2}\right) h_{x}=\frac{\left.w\right|_{z=\varepsilon}}{\varepsilon} .
$$


The N-S equations with free surface are now formulated in a fixed thin domain. Following the approach of T. NisHida et al., we shall compute estimates in Sobolev norms on solutions of the Stokes problem. In order to estimate the nonlinear terms, we shall also write some fundamental Sobolev inequalities in thin domain with a particular attention on the optimal dependence of Sobolev constant with respect to $\varepsilon$. This is done in the next section.

3.1.2. Fundamental inequalities in thin domain. In what follows, we write the classical Poincaré inequalities and Sobolev injections for the thin domain $\Omega=$ $\mathbb{T} \times(0, \varepsilon)$. The resulting inequalities are summarized in the following lemma.

Lemma 1. Assume that $\left.u\right|_{z=0}=0$ or $\left.u\right|_{z=\varepsilon}=0$, there exists a constant $C$ independent of $\varepsilon$ so that the following estimates hold

$$
\begin{aligned}
& \|u\|_{0} \leq C \varepsilon\left\|\partial_{z} u\right\|_{0}, \quad\|u\|_{L^{6}(\Omega)} \leq C\|\nabla u\|_{0} \\
& \|u\|_{L^{\infty}(\Omega)} \leq C \sqrt{\varepsilon}\left(\left\|\partial_{z} u\right\|_{0}+\left\|\partial_{z} u_{x}\right\|_{0}\right)
\end{aligned}
$$

In the general case, these inequalities read:

$$
\|u\|_{L^{6}(\Omega)} \leq C \varepsilon^{-\frac{1}{3}}\|u\|_{1}, \quad\|u\|_{L^{\infty}(\Omega)} \leq C \varepsilon^{-\frac{1}{2}}\|u\|_{2},
$$

For a proof of these Sobolev-type inequalities, see the paper of R. Temam and M. ZIANE [13] on Navier-Stokes equations in thin domain. Those inequalities are deduced from Poincaré inequalities (Proposition 2.1), Agmon's inequalities in thin domain (Proposition 2.2) and anisotropic Ladhyzhenskaya's inequality (Proposition 2.3). A straightforward application of this lemma yields the further Sobolev-type inequalities. We easily prove the following result.

Lemma 2. Denote $H_{0}^{s}$ the subspace of Sobolev space $H^{s}(\Omega)$ vanishing either on $z=0$ or $z=\epsilon$. There exists a constant $C$, independent of $\varepsilon$ so that the following inequalities hold true:

$$
\begin{aligned}
& \|u v\|_{0} \leq C \sqrt{\varepsilon}\left(\|\nabla u\|_{0}+\left\|\nabla u_{x}\right\|_{0}\right)\|v\|_{0}, \quad \forall(u, v) \in H_{0}^{2} \times L^{2}, \\
& \|u v\|_{0} \leq C \varepsilon^{-\frac{1}{2}}\|u\|_{2}\|v\|_{0}, \quad \forall(u, v) \in H^{2} \times L^{2},
\end{aligned}
$$

Moreover, we can also prove that

$$
\begin{aligned}
& \|u v\|_{0} \leq C \varepsilon^{\frac{1}{6}}\|\nabla u\|_{0}\|\nabla v\|_{0}, \quad \forall(u, v) \in H_{0}^{1} \times H_{0}^{1}, \\
& \|u v\|_{0} \leq C \varepsilon^{-\frac{1}{6}}\|\nabla u\|_{0}\|v\|_{1}, \quad \forall(u, v) \in H_{0}^{1} \times H^{1}, \\
& \|u v\|_{0} \leq C \varepsilon^{-\frac{1}{2}}\|u\|_{1}\|v\|_{1}, \quad \forall(u, v) \in H^{1} \times H^{1} .
\end{aligned}
$$

The proof of the first set of inequalities is obtained using the classical Sobolev embedding $H^{2}(\Omega) \hookrightarrow L^{\infty}(\Omega)$, with the optimal Sobolev constant defined in Lemma 1 . The second set of inequalities results from the embedding $L^{6}(\Omega) \times L^{6}(\Omega) \hookrightarrow L^{2}(\Omega)$ together with the estimate

$$
\|u v\|_{L^{2}(\Omega)} \leq C \varepsilon^{\frac{1}{6}}\|u\|_{L^{6}(\Omega)}\|v\|_{L^{6}(\Omega)},
$$

with $C$ is independent of $\varepsilon$. 
Applying Lemma 1, we can also obtain estimates of the extension $\bar{h}$ of $h$ on the domain $\Omega$. The proof of the following lemma is straightforward.

Lemma 3. There exists a constant $C$ independent of $\varepsilon$ such that for any $\alpha=$ $\left(\alpha_{1}, \alpha_{2}\right)$ and $n=|\alpha|$, the extension $\bar{h}$ of $h$ on $\Omega$ satisfies the estimates

$$
\left\|\partial_{j}^{\alpha} \bar{h}\right\|_{0} \leq C \sqrt{\varepsilon}\left|\partial_{x}^{n} h\right|_{0}, \quad\left\|\partial_{j}^{\alpha} \bar{h}\right\|_{0} \leq C|h|_{n-\frac{1}{2}}
$$

Moreover, using the fact that $\partial_{z}^{2 k+1} \bar{h}(x, \epsilon)=0$ and Lemma 1, one obtains the uniform estimates:

$$
\|\bar{h}\|_{\infty} \leq C\left|h_{x}\right|_{0}, \quad\left\|\bar{h}_{z}\right\|_{\infty} \leq C \sqrt{\varepsilon}|h|_{\frac{5}{2}}, \quad\left\|\bar{h}_{z}\right\|_{0} \leq C \varepsilon\left|h_{x x}\right|_{0} .
$$

Proof. We prove the estimates on $\left\|\partial_{j}^{\alpha} \bar{h}\right\|_{0}$ and start with the derivatives in the $x$-direction. It is an easy computation to show that

$$
\left\|\partial_{x}^{n} \bar{h}\right\|_{0}^{2}=\sum_{k \neq 0}\left|h_{k}\right|^{2}|k|^{2 n} \int_{0}^{\varepsilon} \frac{d z}{\left(1+k^{2}(z-\varepsilon)^{2}\right)^{2}} .
$$

With a change of variable in (62), one proves that

$$
\left\|\partial_{x}^{n} \bar{h}\right\|_{0}^{2}=\sum_{k \neq 0}\left|h_{k}\right|^{2}|k|^{2 n-1} f(\varepsilon|k|), \quad f(x)=\int_{0}^{x} \frac{d u}{\left(1+u^{2}\right)^{2}} .
$$

The function $f$ is bounded and $|f(x)| \leq x, \forall x \in(0,+\infty)$. We easily prove that

$$
\left\|\partial_{x}^{n} \bar{h}\right\|_{0} \leq \varepsilon\left|\partial_{x}^{n} h\right|_{0}, \quad\left\|\partial_{x}^{n} \bar{h}\right\|_{0} \leq\|f\|_{\infty}|h|_{n-\frac{1}{2}} .
$$

In a similar way, one can prove that

$$
\left\|\partial_{x}^{n} \partial_{z} \bar{h}\right\|_{0}^{2}=\sum_{k \neq 0}\left|h_{k}\right|^{2}|k|^{2 n+1} g(\varepsilon|k|), \quad g(x)=\int_{0}^{x} \frac{u^{2} d u}{\left(1+u^{2}\right)^{4}}
$$

The function $g$ is bounded and $|g(x)| \leq x, \forall x \in(0,+\infty)$. We easily deduce the estimates on $\left\|\partial_{x}^{n} \partial_{z} \bar{h}\right\|_{0}$. The bounds on the higher order derivatives of $\tilde{h}$ follows similarly.

Following the method of T. NISHIDA et al. [12] to obtain a priori estimates on the solutions of the full Navier-Stokes equations, we shall write energy inequalities involving the fluid velocity $(u, w)$, the time and transverse derivatives $\partial_{t}^{\alpha} \partial_{x}^{\beta}(u, w)$ and their gradients. We estimate the other derivatives of $(u, w)$ and the pressure $p$ using the regularity in the Stokes problem. The main issue here is to obtain the dependence of the regularity constant with respect to $\varepsilon$. In what follows, we consider the Stokes problem on the thin domain $\Omega=\mathbb{T} \times(0, \varepsilon)$ with Dirichlet boundary conditions. We shall prove the following result. $\Omega$ :

Lemma 4. Let $f \in\left(H^{1}(\Omega)\right)^{2}, \phi \in\left(H^{\frac{3}{2}}(\mathbb{T})\right)^{2}$ and consider the Stokes problem in

$$
-\Delta \bar{u}+\nabla p=f,
$$

$$
\operatorname{div} \bar{u}=0,
$$


with the boundary conditions

$$
\left.\bar{u}\right|_{z=0}=0,\left.\quad \bar{u}\right|_{z=\varepsilon}=\phi .
$$

There exists a constant $C$ independent of $\varepsilon$ so that for $i=0,1$ :

$$
\|\bar{u}\|_{2+i}+\|\nabla p\|_{i} \leq C\left(\|f\|_{i}+\frac{\left|\phi_{1}\right|_{1+i}}{\varepsilon^{\frac{3}{2}}}+\frac{\left|\phi_{2}\right|_{i}}{\varepsilon^{\frac{5}{2}}}+\left\|\nabla \bar{u}_{x}\right\|_{0}+\left\|\nabla \bar{u}_{x x}\right\|_{0}\right) .
$$

Proof. We first prove the case $i=0$. More precisely, we show that there exists a constant $C$ independent of $\varepsilon$ so that:

$$
\|\bar{u}\|_{2}+\|\nabla p\|_{0} \leq C\left(\|f\|_{0}+\frac{\left|\phi_{1}\right|_{1}}{\varepsilon^{\frac{3}{2}}}+\frac{\left|\phi_{2}\right|_{0}}{\varepsilon^{\frac{5}{2}}}+\left\|\nabla \bar{u}_{x}\right\|_{0}\right) .
$$

The case $i=1$ follows immediately from that particular case: we derive the Stokes equations in the $x$-direction and use the case $i=0$ to estimate the functions $\partial_{x}^{\alpha} \partial_{z}^{\beta} \bar{u}, \partial_{x} \nabla p$ for any $\alpha \geq 1, \alpha+\beta \leq 3$. The other derivatives of $w$ and $\partial_{z}^{2} p$ are estimated using the divergence free condition whereas the derivatives of $u$ are estimated using the Stokes equations and in particular

$$
u_{z z}=-f_{1}+\partial_{x} p-u_{x x}
$$

Now we prove (64) and write the function $\bar{u}$ in the form $\bar{u}=(u, w)$. In the sequel, we shall note $C$ a constant independent of $\varepsilon$. Using the Poincaré inequality and the divergence free condition, we easily prove that

$$
\|u\|_{0}+\|w\|_{0}+\|\nabla u\|_{0}+\|\nabla w\|_{0} \leq C\left(\left\|\partial_{z} u\right\|_{0}+\left\|\nabla u_{x}\right\|_{0}\right) .
$$

From the Stokes equation, we deduce that

$$
\left\|\bar{u}_{z z}\right\|_{0} \leq C\left(\|f\|_{0}+\|\nabla p\|_{0}+\left\|\nabla \bar{u}_{x}\right\|_{0}\right) .
$$

Assume for the moment that $\nabla p$ satisfies the estimate

$$
\|\nabla p\|_{0} \leq C\left(\|f\|_{0}+\frac{\left|\phi_{1}\right|_{1}}{\varepsilon^{\frac{3}{2}}}+\frac{\left|\phi_{2}\right|_{0}}{\varepsilon^{\frac{5}{2}}}\right) .
$$

We want to estimate $\left\|\partial_{z} u\right\|_{0}$. An integration by parts yields

$$
\int_{\Omega}\left(\partial_{z} u\right)^{2}=\int_{\mathbb{T}} u(\cdot, \varepsilon) u_{z}(\cdot, \varepsilon) d x-\int_{\Omega} u u_{z z} .
$$

Using the boundary conditions and Cauchy Schwarz inequalities, one finds

$$
\int_{\Omega}\left(\partial_{z} u\right)^{2} \leq\left|\phi_{1}\right|_{0}\left|u_{z}(\cdot, \varepsilon)\right|_{0}+\varepsilon\left\|\partial_{z} u\right\|_{0}\left\|u_{z z}\right\|_{0} .
$$

It is easily proved that

$$
\left|u_{z}(\varepsilon, .)\right|_{0} \leq C\left(\frac{1}{\sqrt{\varepsilon}}\left\|\partial_{z} u\right\|_{0}+\sqrt{\varepsilon}\left\|u_{z z}\right\|_{0}\right) .
$$

Using Young's inequality and the estimate $(65)$ on $u_{z z}$, we obtain

$$
\left\|\partial_{z} u\right\|_{0} \leq C\left(\|f\|_{0}+\frac{\left|\phi_{1}\right|_{0}}{\varepsilon^{\frac{3}{2}}}+\frac{\left|\phi_{2}\right|_{0}}{\varepsilon^{\frac{5}{2}}}\right) .
$$


We prove the estimate (66) on $\nabla p$. From the Stokes equation and the divergence free condition, we easily prove that

$$
\left\|\partial_{z} p\right\|_{0} \leq\|f\|_{0}+\left\|\nabla \bar{u}_{x}\right\|_{0}
$$

Let $\bar{v} \in H^{2}(\Omega)$ so that

$$
\left.\bar{v}\right|_{z=0}=0,\left.\quad \bar{v}\right|_{z=\varepsilon}=\phi, \quad \operatorname{div} \bar{v}=0 .
$$

Such a function exists due to potential theory [7]. Let us write $\bar{u}$ in the form $\bar{u}=\bar{v}+\tilde{u}$. Then we find that $\tilde{u}, p$ satisfies the Stokes problem

$$
\begin{aligned}
& -\Delta \tilde{u}+\nabla p=f+\Delta \bar{v}, \\
& \operatorname{div} \tilde{u}=0,\left.\quad \tilde{u}\right|_{z=0}=\left.\tilde{u}\right|_{z=\varepsilon}=0 .
\end{aligned}
$$

With a symmetry argument, R. TEMAm and M. Ziane [13] (Lemma 2.6) proved that in such a case, there exists $C$ independent of $\varepsilon$ so that

$$
\|\tilde{u}\|_{2}+\|\nabla p\|_{0} \leq C\left(\|f+\Delta \tilde{v}\|_{0}\right)
$$

The estimates obtained in [7] for $\tilde{v}$ would give

$$
\|\bar{u}\|_{2}+\|\nabla p\|_{0} \leq C\left(\|f\|_{0}+\frac{\left|\phi_{1}\right|_{\frac{3}{2}}}{\varepsilon^{\frac{3}{2}}}+\frac{\left|\phi_{2}\right|_{\frac{3}{2}}}{\varepsilon^{\frac{5}{2}}}\right) .
$$

The inequality (69) is not optimal here. In the sequel, we "improve" that estimate provided that we add a correction term $\left\|\nabla u_{x}\right\|_{0}$. Using the divergence free condition, the lemma is proved provided that

$$
\min _{u \in \mathbb{X}}\|\Delta u\|_{0} \leq C\left(\frac{\left|\phi_{1}\right|_{1}}{\varepsilon^{\frac{3}{2}}}+\frac{\left|\phi_{2}\right|_{0}}{\varepsilon^{\frac{5}{2}}}\right),
$$

where $\mathbb{X}$ is the functional space

$$
\mathbb{X}=\left\{u \in H^{2}(\Omega):\left.\quad u\right|_{z=0}=0,\left.\quad u\right|_{z=\varepsilon}=\phi_{1}, \quad \int_{0}^{\epsilon} u_{x}(\cdot, z) d z=-\phi_{2}\right\} .
$$

In order to prove (70), we decompose $u$ and $\phi$ into Fourier series:

$$
u(x, z)=\sum_{k \in \mathbb{Z}} u_{k}(z) e^{i k x}, \quad \phi(x)=\sum_{k \in \mathbb{Z}}\left(\phi_{1, k}, \phi_{2, k}\right) e^{i k x} .
$$

Note that $\phi$ shall satisfy the compatibility condition $\phi_{2,0}=0$. Then the problem reduces to find the optimal solution $u_{k}, k \in \mathbb{Z}$ so that

$$
\int_{0}^{\varepsilon}\left|u_{k}^{\prime \prime}(z)-k^{2} u_{k}(z)\right|^{2} d z=\min \left(\int_{0}^{\varepsilon}\left|v^{\prime \prime}(z)-k^{2} v(z)\right|^{2} d z\right),
$$

under the constraints

$$
\left.v\right|_{z=0}=0,\left.\quad v\right|_{z=\varepsilon}=\phi_{1, k}, \quad \int_{0}^{\varepsilon} v(z) d z=\frac{i \phi_{2, k}}{k} .
$$


It is easily proved that if $u_{k}$ is a solution of the minimization problem, then for any $\phi \in C_{c}^{\infty}(0, \varepsilon)$, the function $u_{k}$ satisfies the Euler-Lagrange equation

$$
\int_{0}^{\varepsilon}\left(u_{k}^{(4)}-2 k^{2} u_{k}^{(2)}+k^{4} u_{k}\right)^{\prime} \phi(z) d z=0 .
$$

As a consequence, the function $u_{k}$ satisfies $u_{k}^{(5)}-2 k^{2} u_{k}^{(3)}+k^{4} u_{k}^{\prime}=0$ and can be written

$$
u_{k}(z)=A+B \cosh (k z)+C \sinh (k z)+D z \cosh (k z)+E z \sinh (k z) .
$$

The minimization problem then reduces to finding $\left(A_{k}, \ldots, E_{k}\right)$ so that

$$
\begin{aligned}
\int_{0}^{\varepsilon} \mid k^{2} A_{k}-2 k\left(D_{k} \sinh (k z)\right. & \left.+E_{k} \cosh (k z)\right)\left.\right|^{2} \\
& =\min \int_{0}^{\varepsilon}\left|k^{2} A-2 k(D \sinh (k z)+E \cosh (k z))\right|^{2},
\end{aligned}
$$

whereas the constraints (71) read

$$
\begin{aligned}
& A+B=0, \\
& A+B \cosh (k \varepsilon)+C \sinh (k \varepsilon)+D \varepsilon \cosh (k \varepsilon)+E \varepsilon \sinh (k \varepsilon)=\phi_{1, k}, \\
& \varepsilon A+B \frac{\sinh (k \varepsilon)}{k}+C \frac{\cosh (k \varepsilon)-1}{k} \\
& +D \frac{1-\cosh (k \varepsilon)+\varepsilon k \sinh (k \varepsilon)}{k^{2}}+E \frac{\varepsilon k \cosh (k \varepsilon)-\sinh (k \varepsilon)}{k^{2}}=\frac{i \phi_{2, k}}{k} .
\end{aligned}
$$

We eliminate $A$ from the constraints (72). Furthermore, we can consider, without loss of generality, the minimization problem in $\mathbb{R}$ :

$$
\begin{aligned}
\int_{0}^{\varepsilon} \mid k^{2} B_{k}+2 k\left(D_{k} \sinh (k z)\right. & \left.+E_{k} \cosh (k z)\right)\left.\right|^{2} \\
& =\min \int_{0}^{\varepsilon}\left|k^{2} B+2 k(D \sinh (k z)+E \cosh (k z))\right|^{2},
\end{aligned}
$$

under the constraints

$$
\begin{aligned}
& B(\cosh (k \varepsilon)-1)+C \sinh (k \varepsilon)+D \varepsilon \cosh (k \varepsilon)+E \varepsilon \sinh (k \varepsilon)=\phi_{1, k}, \\
& B(\sinh (k \varepsilon)-k \varepsilon)+C(\cosh (k \varepsilon)-1) \\
& \quad+D\left(\varepsilon \sinh (k \varepsilon)-\frac{\cosh (k \varepsilon)-1}{k}\right)+E\left(\varepsilon \cosh (k \varepsilon)-\frac{\sinh (k \varepsilon)}{k}\right)=\phi_{2, k},
\end{aligned}
$$

where $\phi_{i, k}, A_{k}, \ldots, E_{k} \in \mathbb{R}$. Here, we choose $D=E=0$. In that case, we find

$$
B=\frac{\cosh (k \varepsilon)-1}{2(1-\cosh (k \varepsilon))+k \varepsilon \sinh (k \varepsilon)} \phi_{1, k}+\frac{\sinh (k \varepsilon)}{2(1-\cosh (k \varepsilon))+k \varepsilon \sinh (k \varepsilon)} \phi_{2, k} .
$$

In order to simplify the notations, we introduce $A_{1}^{k}, A_{2}^{k}$ so that

$$
B=A_{1}^{k} \phi_{1, k}+A_{2}^{k} \phi_{2, k}
$$


and we note $u(x, z)=\sum_{k \in \mathbb{Z}} u_{k}(z) e^{i k x}$ the solution associated to that Fourier decomposition, we find that

$$
\|\Delta u\|_{0} \leq \sum_{k} \varepsilon|k|^{4}\left(\left(A_{1}^{k}\right)^{2}\left|\phi_{1, k}\right|^{2}+\left(A_{2}^{k}\right)^{2}\left|\phi_{2, k}\right|^{2}\right) .
$$

We estimate $\sum_{k} \varepsilon|k|^{4}\left(A_{1}^{k}\right)^{2}\left|\phi_{1, k}\right|^{2}$ : separating the different cases $k \varepsilon=\mathcal{O}(\varepsilon), k \varepsilon=$ $\mathcal{O}(1)$ and $k \varepsilon \gg 1$, we obtain

$$
\sum_{k} \varepsilon|k|^{4}\left(A_{1}^{k}\right)^{2}\left|\phi_{1, k}\right|^{2} \leq C \frac{\left|\phi_{1}\right|_{1}^{2}}{\varepsilon^{3}}
$$

with $C$, a constant independent of $\varepsilon$. Similarly, we prove that

$$
\sum_{k} \varepsilon|k|^{4}\left(A_{2}^{k}\right)^{2}\left|\phi_{2, k}\right|^{2} \leq C \frac{\left|\phi_{2}\right|_{0}^{2}}{\varepsilon^{5}}
$$

As a consequence, we have proved that

$$
\|\nabla p\|_{0} \leq C\left(\|f\|_{0}+\frac{\left|\phi_{1}\right|_{1}}{\varepsilon^{\frac{3}{2}}}+\frac{\left|\phi_{2}\right|_{0}}{\varepsilon^{\frac{5}{2}}}\right) .
$$

This completes the proof of the lemma.

We shall also give a formulation of a Körn's inequality in the thin domain setting. H. ITO has proved, in [10], the following result

LEMma 5. Let $u \in\left(H_{1}(\Omega)\right)^{2}$ so that

$$
\left.u\right|_{z=0}=0, \quad \operatorname{div} u=0 .
$$

Then the following Körn's inequality holds

$$
\|D(u)\|_{L^{2}(\Omega)}^{2}=\left\|\frac{\nabla u+\nabla u^{t}}{2}\right\|_{L^{2}(\Omega)}^{2} \geq \frac{2}{3}\|\nabla u\|_{L^{2}(\Omega)}^{2} .
$$

3.2. Classical estimates in the shallow water scaling. In the following, we shall prove that the energy estimates obtained by T. NISHIDA and coauthors in [12] still hold true in the shallow water scaling. We recall the Navier-Stokes system, written in the fixed domain $\Omega=\mathbb{T} \times(0, \varepsilon)$.

$$
\begin{aligned}
& u_{t}+U u_{x}+U_{z} w+2 \mu p_{x}=\mu \Delta u+F_{1}, \\
& w_{t}+U w_{x}+2 \mu p_{z}=\mu \Delta w+F_{2}, \\
& u_{x}+w_{z}=0
\end{aligned}
$$

The continuity of the fluid stress at the fixed boundary $z=\varepsilon$ reads in $\mathbb{T}$,

$$
\begin{aligned}
& \left.u_{z}\right|_{z=\varepsilon}+\left.w_{x}\right|_{z=\varepsilon}-2 \frac{s}{\varepsilon} h=H_{1}, \\
& \left.P\right|_{z=\varepsilon}-\frac{c}{\varepsilon} h+\varepsilon W h_{x x}+\left.u_{x}\right|_{z=\varepsilon}=H_{2},
\end{aligned}
$$


whereas $h$ satisfies the equation in $\mathbb{T}$ :

$$
h_{t}+s\left(1-h^{2}\right) h_{x}=\frac{\left.w\right|_{z=\varepsilon}}{\varepsilon} .
$$

We recall that the full expressions of the nonlinear terms $F_{1}, F_{2}$ in the Navier-Stokes equations and $H_{1}, H_{2}$ in the boundary conditions are given by $(58),(60)$. The nondimensional constant $\mu$ is given by $\mu=\varepsilon /$ Re. In what follows, we shall denote $\beta(h)$ the symmetric matrix $\beta(h)=b(h)(I d-b(h))^{-1}$ with

$$
b(h)=\left(\begin{array}{ll}
-\partial_{z}(z h) & z \partial_{x} h \\
z \partial_{x} h & \frac{\partial_{z}(z h)-\left(z \partial_{x} h\right)^{2}}{1+\partial_{z}(z h)}
\end{array}\right) .
$$

The purpose of that section is to prove the following result.

THEOREM 1. There exists $\varepsilon_{1}>0$ such that if

$$
\frac{\sin (\theta)}{\sqrt{\varepsilon} \kappa} \leq \varepsilon_{1}, \quad \frac{\left|h_{0}\right|_{\infty}}{\varepsilon}+\left|\partial_{x}^{2} h_{0}\right|_{0}+\varepsilon^{\frac{1}{4}}\left|h_{0}\right|_{\frac{5}{2}}+\sqrt{\varepsilon}\left\|\left(u_{0}, w_{0}\right)\right\|_{H^{2}} \leq \varepsilon_{1},
$$

then there exists $\gamma_{1}$, so that the following energy estimate holds

$$
\frac{\partial}{\partial t}(E(u, w)+\mu K(h))+\gamma_{1} \mu G(u, w) \leq 0 .
$$

Here $E, K, G$ denote the functions

$$
\begin{aligned}
E(u, w) & =\sum_{j=0}^{2}\left\|\partial_{x}^{j}(u, w)\right\|_{0}+\left((I d+\beta(h)) \partial_{t} u, \partial_{t} u\right), \\
K(h) & =c\left(\sum_{j=0}^{2}\left|\partial_{x}^{j} h\right|_{0}+\left|\partial_{t} h\right|_{0}^{2}\right)+\kappa\left(\sum_{j=0}^{2}\left|\partial_{x}^{j+1} h\right|_{0}+\left|\partial_{x t} h\right|_{0}^{2}\right) \\
G(u, w) & =\sum_{j=0}^{2}\left\|\nabla \partial_{x}^{j}(u, w)\right\|_{0}^{2}+\left\|\nabla \partial_{t}(u, w)\right\|_{0}^{2} .
\end{aligned}
$$

REMARK. As we shall see later, this estimate is sufficient to obtain estimates of the fluid speed $(u, w)$ in the classical Sobolev spaces $H^{2}(\Omega), H^{3}(\Omega)$.

Proof. Following the paper of T. NisHIDA and coauthors [12], we shall decompose the proof into several steps. First, we show the following result.

Proposition 1. There exists $\varepsilon_{1}>0$ so that for

$$
\left|h_{x x}^{0}\right|_{0}+\varepsilon^{\frac{1}{3}}\left|h^{0}\right|_{\frac{5}{2}}+\sqrt{\varepsilon}\left\|\left(u^{0}, w^{0}\right)\right\|_{2} \leq \varepsilon_{1},
$$

the solution $(u, w), \nabla p$ of Navier-Stokes system satisfies for $i=0,1$

$$
\|\bar{u}\|_{2+i}+\|\nabla p\|_{i} \leq \frac{C}{\mu}\left(\left\|\bar{u}_{t}\right\|_{i}+\left(s+\mathbf{R e}^{-1}\right)\left(\|\nabla \bar{u}\|_{0}+\left\|\nabla \bar{u}_{x}\right\|_{0}+\left\|\nabla \bar{u}_{x x}\right\|_{0}\right)\right) .
$$

In order to prove that proposition, we consider the Stokes problem 


$$
\begin{aligned}
& -\mu \Delta \bar{u}+2 \mu \nabla p=F-\bar{u}_{t}-U \bar{u}_{x}-U^{\prime}(w, 0)^{t} \\
& \operatorname{div} \bar{u}=0, \\
& \left.\bar{u}\right|_{z=0}=0,\left.\quad \bar{u}\right|_{z=\varepsilon}=\left(\left.u\right|_{z=\varepsilon},\left.w\right|_{z=\varepsilon}\right) .
\end{aligned}
$$

We apply Lemma 4 to the Stokes problem (79) and obtain

$$
\mu\|\bar{u}\|_{2+i}+2 \mu\|\nabla p\|_{i} \leq C\left(\left\|F-\bar{u}_{t}-U \bar{u}_{x}-U^{\prime}(w, 0)^{t}\right\|_{i}+\mu\left(\frac{\left.|u|_{z=\varepsilon}\right|_{1+i}}{\varepsilon^{\frac{3}{2}}}+\frac{\left.|w|_{z=\varepsilon}\right|_{i}}{\varepsilon^{\frac{5}{2}}}\right)\right) .
$$

The boundary terms are bounded by

$$
\begin{aligned}
& \left.|u|_{z=\varepsilon}\right|_{1+i} \leq \sqrt{\varepsilon}\left(\left\|\nabla u_{x}\right\|_{0}+\left\|\nabla u_{x x}\right\|_{0}\right), \\
& \left.|w|_{z=\varepsilon}\right|_{i} \leq \varepsilon \sqrt{\varepsilon}\left(\left\|\nabla u_{x}\right\|_{0}+\left\|\nabla u_{x x}\right\|_{0}\right) .
\end{aligned}
$$

We obtain the second inequality using the divergence free condition in the form $w_{z}=$ $-u_{x}$ and a Poincaré inequality. Similarly, the linear term $U \bar{u}_{x}+U^{\prime}(w, 0)^{t}$ satisfies for $i=0,1$

$$
\left\|U \bar{u}_{x}+U^{\prime}(w, 0)^{t}\right\|_{i} \leq C s\left(\|\nabla \bar{u}\|_{0}+\left\|\nabla \bar{u}_{x}\right\|_{0}+\left\|\nabla \bar{u}_{x x}\right\|_{0}\right),
$$

whereas $\left\|\bar{u}_{t}\right\|_{i} \leq C\left\|\nabla \bar{u}_{t}\right\|_{0}$. Inserting those inequalities into (80), we obtain

$$
\begin{aligned}
& \mu\|u\|_{2+i}+2 \mu\|\nabla p\|_{i} \leq \\
& C\left(\|F\|_{i}+\left\|\nabla \bar{u}_{t}\right\|_{0}+\left(s+\mathbf{R e}^{-1}\right)\left(\|\nabla \bar{u}\|_{0}+\left\|\nabla \bar{u}_{x}\right\|_{0}+\left\|\nabla \bar{u}_{x x}\right\|_{0}\right)\right) .
\end{aligned}
$$

Now we consider the nonlinear terms $F=\left(F_{1}, F_{2}\right)$. We can write $F$ in the form $F=\mu b(h) \nabla p+\widetilde{F}$ where $\widetilde{F}$ contains only derivatives of $h$ and $\bar{u}$. We easily prove that

$$
\|\mu b(h) \nabla p\|_{i} \leq C\left(\left|h_{x x}\right|_{0}+\varepsilon^{\frac{1}{3}}|h|_{\frac{5}{2}}\right)\|\nabla p\|_{i} .
$$

Under the hypothesis of the proposition and for $\varepsilon_{1}$ sufficiently small, the inequality (81) still holds with $F$ replaced by $\widetilde{F}$.

The second order terms of $\widetilde{F}$ have the form $\mu a_{j, k}(h, \nabla h) \partial_{z}^{j} \partial_{x}^{k} \bar{u}$ with $j+k=2$ and are estimated by

$$
\left\|\mu a_{j, k}(h, \nabla h) \partial_{z}^{j} \partial_{x}^{k} \bar{u}\right\|_{i} \leq \mu C\left(\varepsilon_{1}\right)\left(\left|h_{x x}\right|+\varepsilon^{\frac{1}{3}}|h|_{\frac{5}{2}}\right)\|\bar{u}\|_{2+i} .
$$

Next, we consider the nonlinear terms involving a third order derivative in $h$. More precisely, those terms have the form $\mu b_{j, k}(h, \nabla h, z) u \partial_{x}^{j} \partial_{z}^{k} h$ with $j+k=3$. We prove easily that

$$
\begin{aligned}
& \left\|b_{j, k}(h, \nabla h, z) u \partial_{x}^{j} \partial_{z}^{k} h\right\|_{0} \leq C\left(\varepsilon_{1}\right) \varepsilon^{\frac{3}{2}}|h|_{\frac{5}{2}}\left(\|\nabla u\|_{0}+\left\|\nabla u_{x}\right\|_{0}\right), \\
& \left\|b_{j, k}(h, \nabla h, z) u \partial_{x}^{j} \partial_{z}^{k} h\right\|_{1} \leq C\left(\varepsilon_{1}\right) \sqrt{\varepsilon}\|u\|_{2}|h|_{\frac{7}{2}} .
\end{aligned}
$$

We shall obtain a bound for $|h|_{\frac{7}{2}}$ : for that purpose, we consider the equation on the boundary $z=\varepsilon$ :

$$
\left.p\right|_{z=\varepsilon}-\frac{c}{\varepsilon} h+\varepsilon W h_{x x}+\left.u_{x}\right|_{z=\varepsilon}=H_{2} .
$$


We apply the operator $\partial_{x}^{\frac{3}{2}}$ to $(83)$ and take the scalar product with $\partial_{x}^{\frac{7}{2}} h$. Using Cauchy Schwarz inequality, we obtain

$$
\frac{c}{\varepsilon}|h|_{\frac{5}{2}}^{2}+\varepsilon W|h|_{\frac{7}{2}}^{2} \leq|h|_{\frac{7}{2}}\left(\left|\partial_{x}^{\frac{3}{2}} p\right|_{0}+\left|\partial_{x}^{\frac{5}{2}} u(\varepsilon, .)\right|_{0}+\left|\partial_{x}^{\frac{3}{2}} H_{2}\right|_{0}\right)
$$

We set $\kappa=\varepsilon^{2} W$. In the derivation of the shallow water model, we assumed that $\kappa=\mathcal{O}(1)$ : in particular, $\kappa$ can be set to 0 . In what follows, we assume for the moment that $\kappa>0$. We deduce from (84) that

$$
\kappa|h|_{\frac{7}{2}} \leq C \varepsilon\left(\|\nabla p\|_{1}+\left\|\nabla u_{x x}\right\|_{0}+\left|\partial_{x}^{\frac{3}{2}} H_{2}\right|_{0}\right) .
$$

The Sobolev space $H^{\frac{3}{2}}(\mathbb{T})$ being an algebra, we easily prove that

$$
\left|\partial_{x}^{\frac{3}{2}} H_{2}\right|_{0} \leq C\left(\left\|\nabla u_{x}\right\|_{0}+\left\|\nabla u_{x x}\right\|_{0}+\varepsilon\|u\|_{3}\right)+C \varepsilon^{2}\left(\|u\|_{2}+|h|_{\frac{5}{2}}\right)|h|_{\frac{5}{2}}|h|_{\frac{7}{2}} .
$$

Inserting that inequality into (85), one obtains

$$
\left(\kappa-C \varepsilon^{3}\left(\|u\|_{2}+|h|_{\frac{5}{2}}\right)|h|_{\frac{5}{2}}\right)|h|_{\frac{7}{2}} \leq C \varepsilon\left(\|\nabla p\|_{1}+\varepsilon\|u\|_{3}+\left\|\nabla u_{x}\right\|_{0}+\left\|\nabla u_{x x}\right\|_{0}\right) .
$$

Substituting (86) into (82), we easily obtain that for $\varepsilon_{1}$ sufficiently small, those terms have no influence in (78). Furthermore, the other nonlinear terms are of lower order and have no influence here. The proof of Proposition 1 is then complete.

We are now in a position to obtain energy estimates: according to Proposition 1, we shall derive estimates for $\partial_{x}^{\alpha} u$ with $\alpha=0,1,2$ and $\partial_{t} u$ and their gradients. We first consider the derivatives in the $x$-direction. We note $\gamma$ a constant defined by $\gamma=$ $\frac{2}{3}-2 s$ Re. In the following, we shall denote $\|u\|=\|u\|_{L^{2}(\Omega)},((u, v))=(u, v)_{\left(L^{2}(\Omega)\right)^{2}}$ and $|h|=|h|_{L^{2}(\mathbb{T})}$. One can prove the following result. satisfy

Proposition 2. Setting $\bar{u}=(u, w)$, the fluid velocity $\bar{u}$ and the fluid height $h$

$$
\begin{gathered}
\partial_{t}\left(\frac{\|\bar{u}\|^{2}}{2 \mu}+\left(c|h|^{2}+\kappa\left|h_{x}\right|^{2}\right)\right)+\gamma\|\nabla \bar{u}\|^{2} \leq\left.\frac{2 s}{\varepsilon} \int_{\mathbb{T}} h u\right|_{z=\epsilon} \\
+\left.\int_{\mathbb{T}} H_{1} u\right|_{z=\epsilon}-\left.2 H_{2} w\right|_{z=\epsilon}+2 \kappa s h^{2} h_{x} h_{x x}+\frac{1}{\mu}((F, \bar{u})) \\
\partial_{t}\left(\frac{\left\|\bar{u}_{x}\right\|^{2}}{2 \mu}+\left(c\left|h_{x}\right|^{2}+\kappa\left|h_{x x}\right|^{2}\right)\right)+\gamma\left\|\nabla \bar{u}_{x}\right\|^{2} \leq\left.\frac{2 s}{\varepsilon} \int_{\mathbb{T}} h_{x} u_{x}\right|_{z=\epsilon} \\
+\left.\int_{\mathbb{T}}\left(H_{1}\right)_{x}\right|_{z=\epsilon} u_{x}-\left.2\left(H_{2}\right)_{x} w_{x}\right|_{z=\epsilon}-2 s\left(c h_{x}-\kappa h_{x x x}\right) \partial_{x}\left(h^{2} h_{x}\right)+\frac{1}{\mu}\left(\left(F_{x}, \bar{u}_{x}\right)\right) \\
\partial_{t}\left(\frac{\left\|\bar{u}_{x x}\right\|^{2}}{2 \mu}+\left(c\left|h_{x x}\right|^{2}+\kappa\left|h_{x x x}\right|^{2}\right)\right)+\gamma\left\|\nabla \bar{u}_{x x}\right\|^{2} \leq\left.\frac{2 s}{\varepsilon} \int_{\mathbb{T}} h_{x x} u_{x x}\right|_{z=\epsilon} \\
+\left.\int_{\mathbb{T}}\left(H_{1}\right)_{x x} u_{x x}\right|_{z=\epsilon}-\left.2\left(H_{2}\right)_{x x} w_{x x}\right|_{z=\epsilon}-2 s\left(c h_{x x}-\kappa h_{x x x x}\right) \partial_{x x}\left(h^{2} h_{x}\right)+\frac{1}{\mu}\left(\left(F_{x x}, \bar{u}_{x x}\right)\right) .
\end{gathered}
$$


Denote $F=\widetilde{F}+2 \mu b(h) \nabla p$ and $\beta=b(h)(I d-b(h))^{-1}$. The function $\bar{u}_{t}$ and $h_{t}$ satisfy the estimate

$$
\begin{aligned}
& \partial_{t}\left(\frac{\left((1+\beta(h)) \bar{u}_{t}, \bar{u}_{t}\right)}{2 \mu}+\left(c\left|h_{t}\right|^{2}+\kappa\left|h_{x t}\right|^{2}\right)\right)+\gamma\left\|\nabla \bar{u}_{t}\right\|^{2} \\
& \leq\left.\frac{2 s}{\varepsilon} \int_{\mathbb{T}} h_{t} u_{t}\right|_{z=\epsilon}+\left.\int_{\mathbb{T}}\left(H_{1}\right)_{t} u_{t}\right|_{z=\epsilon}-\left.2\left(H_{2}\right)_{t} w_{t}\right|_{z=\epsilon}-2 s\left(c h_{t}-\kappa h_{x x t}\right) \partial_{t}\left(h^{2} h_{x}\right) \\
& +\frac{1}{\mu}\left(\left(\widetilde{F}_{t}, \bar{u}_{t}\right)\right)+2\left(\left.\partial_{t} b(h) \nabla p\right|_{z=\epsilon},\left.\bar{u}_{t}\right|_{z=\epsilon}\right)+\frac{1}{2}\left(\left.\partial_{t} \beta(h) \bar{u}_{t}\right|_{z=\epsilon},\left.\bar{u}_{t}\right|_{z=\epsilon}\right) \\
& +\frac{1}{\mu}\left(\left.\beta(h)\left(\widetilde{F}_{t}+2 \mu \partial_{t} b \nabla p-U \bar{u}_{x t}-U^{\prime}\left(w_{t}, 0\right)^{t}\right)\right|_{z=\epsilon},\left.\bar{u}_{t}\right|_{z=\epsilon}\right) .
\end{aligned}
$$

Proof. The method for proving (87)- (90) is similar to the one employed by T. NisHIDA and coauthors: see [12] for more details. We shall mention here that we have applied Lemma 5 and used a Körn's inequality

$$
\|D(\bar{u})\|_{\left(L^{2}(\Omega)\right)^{2}}^{2} \geq c(\Omega)\|\nabla \bar{u}\|_{\left(L^{2}(\Omega)\right)^{2}}^{2},
$$

with a constant $c(\Omega)=2 / 3$ that is independent of $\varepsilon$.

Let us prove Theorem 1: for that purpose, we sum the inequalities (87)-(90). We obtain

$$
\frac{\partial}{\partial t}\left(\frac{E(u, w)}{\mu}+K(h)\right)+\gamma G(u, w) \leq \mathcal{R}_{n l}
$$

where $\mathcal{R}_{n l}$ is defined as

$$
\begin{aligned}
\mathcal{R}_{n l} & =\left.\frac{2 s}{\varepsilon} \int_{\mathbb{T}} h u\right|_{z=\epsilon}+\left.h_{x} u_{x}\right|_{z=\epsilon}+\left.h_{x x} u_{x x}\right|_{z=\epsilon}+\left.h_{t} u_{t}\right|_{z=\epsilon} \\
& +\frac{1}{\mu}\left((F, \bar{u})+\left(F_{x}, \bar{u}_{x}\right)+\left(F_{x x}, \bar{u}_{x x}\right)+\left(\widetilde{F}_{t}, \bar{u}_{t}\right)\right) \\
& +\frac{1}{\mu}\left(\left.\beta(h)\left(\widetilde{F}_{t}+2 \mu \partial_{t} b \nabla p-U \bar{u}_{x t}-U^{\prime}\left(w_{t}, 0\right)^{t}\right)\right|_{z=\epsilon},\left.\bar{u}_{t}\right|_{z=\epsilon}\right) \\
& +2\left(\left.\partial_{t} b(h) \nabla p\right|_{z=\epsilon},\left.\bar{u}_{t}\right|_{z=\epsilon}\right)+\frac{1}{2}\left(\left.\partial_{t} \beta(h) \bar{u}_{t}\right|_{z=\epsilon},\left.\bar{u}_{t}\right|_{z=\epsilon}\right) \\
& +\left.\int_{\mathbb{T}} H_{1} u\right|_{z=\epsilon}-\left.2 H_{2} w\right|_{z=\epsilon}+2 \kappa s h^{2} h_{x} h_{x x} \\
& +\left.\int_{\mathbb{T}}\left(H_{1}\right)_{x} u_{x}\right|_{z=\epsilon}-\left.2\left(H_{2}\right)_{x} w_{x}\right|_{z=\epsilon}-2 s\left(c h_{x}-\kappa h_{x x x}\right) \partial_{x}\left(h^{2} h_{x}\right) \\
& +\left.\int_{\mathbb{T}}\left(H_{1}\right)_{x x} u_{x x}\right|_{z=\epsilon}-\left.2\left(H_{2}\right)_{x x} w_{x x}\right|_{z=\epsilon}-2 s\left(c h_{x x}-\kappa h_{x x x x}\right) \partial_{x x}\left(h^{2} h_{x}\right) \\
& +\left.\int_{\mathbb{T}}\left(H_{1}\right)_{t} u_{t}\right|_{z=\epsilon}-\left.2\left(H_{2}\right)_{t} w_{t}\right|_{z=\epsilon}-2 s\left(c h_{t}-\kappa h_{x x t}\right) \partial_{t}\left(h^{2} h_{x}\right) .
\end{aligned}
$$

We show that under the hypothesis (77), and for $\varepsilon_{1} 0$ sufficiently small, $|R|_{n l} \leq$ $\frac{\gamma}{2} G(u, w)$ : that proves Theorem 1 with the choice $\gamma_{1}=\frac{\gamma}{2}$. Denoting $I_{1}$ the singular term in front of $2 s / \varepsilon$, we can prove that

$$
\frac{2 s}{\varepsilon}\left|I_{1}\right| \leq \frac{2 s}{\sqrt{\varepsilon}}\left(\left|h_{x x}\right|+\left|h_{t}\right|\right) G(u, w)^{\frac{1}{2}}
$$


Furthermore, we obtain an estimate on $\left|h_{x x}\right|$ with the boundary condition

$$
\left.\varepsilon p\right|_{z=\varepsilon}-c h+\kappa h_{x x}+\left.\varepsilon u_{x}\right|_{z=\varepsilon}=\varepsilon H_{2} .
$$

Mutiply (93) with $h_{x x}$ and integrate over $\mathbb{T}$ : for $\varepsilon_{1}$ sufficiently small, $\left|h_{x x}\right| \leq$ $\frac{C}{\kappa} G(u, w)^{\frac{1}{2}}$. Similarly, using the boundary condition (76), we prove that $\left|h_{t}\right|$ satisfies the same estimate. As a consequence, we have proved that

$$
\frac{2 s}{\varepsilon}\left|I_{1}\right| \leq C \frac{s}{\kappa \sqrt{\varepsilon}} G(u, w)
$$

and for $\varepsilon_{1}$ small enough, that term is "absorbed" in $\gamma G(u, w)$. We consider the other boundary terms: one can show that

$$
\left|\int_{\mathbb{T}} \partial_{x}^{k} H_{1} \partial_{x}^{k} u\right|_{z=\epsilon} \mid \leq \sqrt{\varepsilon} G(u, w), \quad k=0,1,2 .
$$

The boundary terms $\left.\int_{\mathbb{T}} \partial_{x}^{k} H_{2} \partial_{x}^{k} w\right|_{z=\epsilon},\left.\int_{\mathbb{T}} \partial_{t} H_{1} \partial_{t} u\right|_{z=\epsilon},\left.\int_{\mathbb{T}} \partial_{t} H_{2} \partial_{t} u\right|_{z=\epsilon}$ satisfy the same estimate. We consider $I_{2}=\int_{\mathbb{T}} s \kappa h_{x x x x}\left(h^{2} h_{x}\right)_{x x}$ :

$$
\left|I_{2}\right| \leq s \kappa|h|_{\frac{7}{2}}^{2}|h|_{\frac{5}{2}}^{2} \leq C \frac{s}{\sqrt{\varepsilon} \kappa}\left(\sqrt{\varepsilon}|h|_{\frac{5}{2}}^{2}\right) G(u, w) .
$$

The last inequality is obtained with the estimate (86). The other boundary terms are of lower order and can be bounded similarly.

We finish the proof with the terms involving the nonlinear terms $F$. Using a Poincaré inequality and integration by parts in the $x$-direction, one proves that

$$
\left|I_{3}\right|=\frac{1}{\mu}\left|(F, \bar{u})+\left(F_{x}, \bar{u}_{x}\right)\right| \leq\|F\|_{0}\left(\|\nabla \bar{u}\|_{0}+\left\|\nabla \bar{u}_{x x}\right\|_{0}\right) .
$$

It is a lenghtly but straightforward computation to prove that $\|F\|_{0} \leq C \varepsilon_{1} G(u, w)$ and $I_{3}$ is "absorbed" in the term $\gamma G(u, w)$. We consider the term containing high order derivatives $\left(F_{x x}, \bar{u}_{x x}\right)$ : an integration by parts yields $\frac{1}{\mu}\left(F_{x x}, \bar{u}_{x x}\right)=-\frac{1}{\mu}\left(F_{x}, \bar{u}_{x x x}\right)$. We have to deal with the term $I_{4}=\int_{\Omega}\left(J^{-2}-1\right) u_{z z x} u_{x x x}$ : the other terms are of lower order and can be estimated it is easily proved that

$$
\left|I_{4}\right| \leq C\left(\frac{|h|_{\infty}}{\varepsilon}+\sqrt{\varepsilon}|h|_{\frac{5}{2}}\right)\left(\mu\|u\|_{3}\right)\left\|\nabla u_{x x}\right\|_{0} .
$$

Then for $\varepsilon_{1}$ small enough, $\left|I_{4}\right|$ is absorbed by $\gamma F_{\delta}(u, w)$. Similarly, the only term containing time derivatives that is important here has the form

$$
\begin{aligned}
I_{5} & =\int_{\Omega}\left(J^{-2}-1\right) u_{z z t} u_{t} \\
& =\left.\left.\int_{\mathbb{T}}\left(J^{-2}-1\right) u_{z t}\right|_{z=\epsilon} u_{t}\right|_{z=\epsilon}-\int_{\Omega}\left(\left(J^{-2}-1\right) u_{t}\right)_{z} u_{z t} .
\end{aligned}
$$

We deduce $\left.u_{z t}\right|_{z=\epsilon}$ from the boundary condition (75): deriving the equation on $\left.u_{z}\right|_{z=\epsilon}$ with respect to $t$, one obtains

$$
\left.\left.\int_{\mathbb{T}}\left(J^{-2}-1\right) u_{z t}\right|_{z=\epsilon} u_{t}\right|_{z=\epsilon}=\int_{\mathbb{T}}\left(J^{-2}-1\right)\left(H_{1, t}-\left.w_{x t}\right|_{z=\epsilon}-2 \frac{s}{\varepsilon} h_{t}\right) u_{t} .
$$


Those terms have been treated before and we show that $\left|I_{5}\right| \leq C \varepsilon_{1} G(u, w)$.

As a conclusion, we have proved that for $\varepsilon_{1}$ sufficiently small,

$$
\mathcal{R}_{n l} \leq C \varepsilon_{1} G(u, w) \leq \frac{\gamma}{2} G(u, w) .
$$

This completes the proof of Theorem 1.

4. Rigorous derivation of a shallow water model. In that section, we justify rigorously the derivation of the shallow water model from the Navier-Stokes equations. Recall that the evolution system for the fluid height $h$ and the discharge rate $h v=\int_{0}^{h} v$ can be written, in the shallow water scaling as

$$
\left\{\begin{array}{l}
h_{t}+(h v)_{x}=0, \\
(h v)_{t}+\left(\frac{6}{5} h v^{2}+\frac{c h^{2}}{\mathbf{R e}}-\frac{(2 s)^{2}}{75} h^{5}\right)_{x}-\bar{\kappa} h h_{x x x}=\frac{1}{\varepsilon \mathbf{R e}}\left(2 s h-\frac{3 v}{h}\right)+\widetilde{\mathcal{R}},
\end{array}\right.
$$

with $\widetilde{\mathcal{R}}$ a function of $h,(u, w), p$ and their derivatives. Formally, that function $\widetilde{\mathcal{R}}$ is order $\mathcal{O}\left(\varepsilon\left(1+\mathbf{R e}+\mathbf{R e}^{-1}\right)\right)$ and converges formally to 0 as $\varepsilon \rightarrow 0$. In what follows, we prove that $\widetilde{\mathcal{R}}$ converges to 0 as $\varepsilon \rightarrow 0$ in a suitable functional space. As a byproduct of the method of derivation, we also obtained lubrication models with a single equation on the fluid height $h$ that can be justified rigorously.

4.1. Estimate of the remainder in the shallow water model. In that section, we shall prove that $\lim _{\varepsilon \rightarrow 0} \widetilde{R}=0$ in a suitable functional space: we first recall the definition of $\tilde{\mathcal{R}}$. Let $\tilde{u}, \tilde{w}, \tilde{p}, \tilde{h}$ a solution of the Navier-Stokes system (74),(75),(76). We define $u, w, p, h=1+\tilde{h}$ solution of the Navier-Stokes system (8), (9), (10) that was used to derive shallow-water equations associated to $\tilde{u}, \tilde{w}, \tilde{p}, \tilde{h}$ through the change of variables (43), (48), (56). More precisely, the functions $u, w, p$ reads

$$
\begin{aligned}
\left(\begin{array}{c}
u \\
\varepsilon^{-1} w
\end{array}\right)(t, x, z) & =\left(\begin{array}{c}
U \\
0
\end{array}\right)+\frac{1}{J} D \Theta_{t} \circ \Theta_{t}^{-1}(x, \varepsilon z)\left(\begin{array}{c}
\tilde{u} \\
\tilde{w}
\end{array}\right)\left(t, \Theta_{t}^{-1}(x, \varepsilon z, .)\right), \\
p(t, x, z) & =c(1-z)+\varepsilon \tilde{p}\left(t, \Theta_{t}^{-1}(x, \varepsilon z)\right),
\end{aligned}
$$

with $U(z)=s\left(2 z-z^{2}\right)$ and the function $\widetilde{\mathcal{R}}$ is written

$$
\widetilde{\mathcal{R}}=\mathcal{R}_{1}+\mathcal{R}_{2}+\mathcal{R}_{3} .
$$

The function $\mathcal{R}_{1}$ is defined as

$$
\begin{aligned}
\mathcal{R}_{1} & =-\partial_{x} \mathcal{R}_{1}^{(1)}(t, x)-\partial_{x} \mathcal{R}_{1}^{(2)}(t, x) \\
& +\frac{\varepsilon}{\operatorname{Re}} \partial_{x}\left(\int_{0}^{h(t, x)} u_{x}(t, x, \zeta) d \zeta\right)+\bar{\kappa}\left(\left(1-\left(1+\varepsilon^{2} h_{x}^{2}\right)^{-\frac{3}{2}}\right) h_{x} h_{x x}\right)
\end{aligned}
$$

with

$$
\begin{aligned}
\mathcal{R}_{1}^{(1)}(t, x)= & \int_{0}^{h(t, x)}\left(u^{2}-\left(u^{(0)}\right)^{2}\right)(t, x, \zeta) d \zeta \\
& +\frac{12}{15} v \int_{0}^{h(x, t)} \delta u(x, y, t) d y+\frac{2}{15 h}\left(\int_{0}^{h} \delta u(t, x, \zeta) d y\right)^{2}, \\
\mathcal{R}_{1}^{(2)}(t, x)= & \int_{0}^{h(t, x)}\left(p-p^{(0)}\right)(t, x, \zeta) d \zeta .
\end{aligned}
$$


The function $\mathcal{R}_{2}$ reads

$$
\mathcal{R}_{2}=\partial_{z} \mathcal{R}_{2}^{(1)}(0)-\frac{3}{h^{2}} \int_{0}^{h} \mathcal{R}_{2}^{(1)}
$$

with $\mathcal{R}_{2}^{(1)}$ defined as

$$
\begin{aligned}
\mathcal{R}_{2}^{(1)}= & \frac{\varepsilon}{\mathbf{R e}} \int_{0}^{z} \int_{\zeta}^{h} u_{x x}-u_{x x}^{(0)}-\int_{0}^{z} \int_{\zeta}^{h} \frac{2}{\mathbf{R e}}\left(p-p^{(0)}\right)_{x} \\
& -\frac{\varepsilon z}{\operatorname{Re}}\left(\left(w_{x}(h)+\frac{w_{z}(h)}{1-\varepsilon^{2} h_{x}^{2}}\right)-\left(w_{x}^{(0)}(h)+\frac{w_{z}^{(0)}(h)}{1-\varepsilon^{2} h_{x}^{2}}\right)\right) \\
& +\int_{0}^{z} \int_{\zeta}^{h}\left(u-u^{(0)}\right)_{t}+\left(u u_{x}-u^{(0)} u_{x}^{(0)}\right)+\left(w u_{z}-w^{(0)} u_{z}^{(0)}\right),
\end{aligned}
$$

whereas the function $\mathcal{R}_{3}$ is written

$$
\mathcal{R}_{3}=\frac{s h^{2}}{4} \partial_{x}\left(\int_{0}^{h} u-u^{(0)}\right) .
$$

The purpose of that section is to prove the following result.

THEOREM 2. Assume that the capillary constant $\bar{\kappa}$ has the form $\bar{\kappa}=\frac{\kappa}{\sqrt{\varepsilon}}$. Let us suppose that the initial conditions $\left(\tilde{u}_{0}, \tilde{w}_{0}, \tilde{h}_{0}\right)$ satisfy the assumptions of Theorem 1 and that $\frac{\sin (\theta)}{\kappa}$ is sufficiently small. Then the function $\widetilde{\mathcal{R}}$ satisfies the estimate

$$
\|\widetilde{\mathcal{R}}\|_{L^{2}\left(0, \infty ; L^{2}(\mathbb{T})\right)} \leq C \sqrt{\varepsilon}
$$

with $C$, a constant, that does not depend on $\varepsilon$.

Proof. In what follows, we shall express the remainders $\mathcal{R}_{i}$ as functions of $\tilde{u}, \tilde{w}, \tilde{p}, \tilde{p}$ and use the estimates obtained previously. Let us first note that the functions $u, w, p$ read

$$
\begin{aligned}
& u(t, x, z)=s\left(2 z-z^{2}\right)+\frac{1}{J} \tilde{u}\left(\Theta_{t}^{-1}(x, \varepsilon z)\right)=u^{(0)}-2 s \tilde{h} z+\frac{1}{J} \tilde{u}\left(t, \Theta_{t}^{-1}(x, \varepsilon z)\right), \\
& w(t, x, z)=\varepsilon\left(\tilde{w}+\frac{\bar{z} \tilde{h}_{x}}{J} \tilde{u}\right)\left(t, \Theta_{t}^{-1}(x, \varepsilon z)\right) \\
& p(t, x, z)=p^{(0)}-c \tilde{h}+\varepsilon \tilde{p}\left(t, \Theta_{t}^{-1}(x, \varepsilon z)\right)
\end{aligned}
$$

For the sake of simplicity, denote $\bar{z}=\theta_{t}^{-1}(x, \varepsilon z)$ defined so that $\Theta_{t}^{-1}(x, \varepsilon z)=(x, \bar{z})$ and

$$
\varepsilon z=\bar{z}(1+\tilde{h}(t, x, \bar{z}))
$$

The proof of Theorem 2 is done in three steps. We first prove the following result.

Lemma 6. The function $\mathcal{R}_{3}$ satisfies the estimate:

$$
\left\|\mathcal{R}_{3}\right\|_{L^{2}\left(0, \infty ; L^{2}(\mathbb{T})\right)} \leq C \sqrt{\varepsilon}
$$


Proof. The function $\mathcal{R}_{3}$ can be written in the form

$$
\mathcal{R}_{3}=\frac{s h^{2}}{4} \partial_{x}\left(\int_{0}^{1+\tilde{h}}-2 s \tilde{h} z+\frac{\tilde{u}}{J}\left(x, \theta_{t}^{-1}(t, x, \varepsilon z)\right) d z\right) .
$$

We make the change of variable $\bar{z}(1+\tilde{h}(t, x, \bar{z})=\varepsilon z$ in the integral (103):

$$
\begin{aligned}
\mathcal{R}_{3} & =\frac{s h^{2}}{4} \partial_{x}\left(-s \tilde{h} h^{2}+\frac{1}{\varepsilon} \int_{0}^{\varepsilon} \tilde{u}(t, x, \bar{z}) d \bar{z}\right) \\
& =-\frac{s^{2} h^{3}}{4}(1+2 \tilde{h}) \tilde{h}_{x}+\frac{1}{\varepsilon} \int_{0}^{\varepsilon} \tilde{u}_{x}(t, x, z) d z .
\end{aligned}
$$

Recall that we have obtained an estimate of $\left|h_{x x}\right|_{0}$ in the form

$$
\left|h_{x x}\right|_{0} \leq \frac{C}{\kappa} \sqrt{\varepsilon}\left(\sum_{j=0}^{2}\left\|\nabla \partial_{x}^{j} \tilde{u}\right\|_{0}+\left\|\nabla \tilde{u}_{t}\right\|_{0}\right) .
$$

We deduce from Theorem 1 that $|\tilde{h}|_{L^{\infty}\left(0, \infty ; L^{2}(\mathbb{T})\right)} \leq C$. Furthermore, using Poincaré inequality, one finds

$$
\left|\frac{1}{\varepsilon} \int_{0}^{\varepsilon} \tilde{u}_{x}\right|_{0} \leq \sqrt{\varepsilon}\left\|\nabla \tilde{u}_{x}\right\|_{0}
$$

As a consequence, the function $\mathcal{R}_{3}$ satisfies $\left\|\mathcal{R}_{3}\right\|_{L^{2}\left(0, \infty ; L^{2}(\mathbb{T})\right)} \leq C \sqrt{\varepsilon}$. This completes the proof of the lemma. $\square$

We next show that the correction terms obtained from the averaged convection terms and pressure terms are order $\mathcal{O}(\sqrt{\varepsilon})$.

Lemma 7. The function $\mathcal{R}_{1}$ satisfies the estimate:

$$
\left\|\mathcal{R}_{1}\right\|_{L^{2}\left(0, \infty ; L^{2}(\mathbb{T})\right)} \leq C \sqrt{\varepsilon}
$$

Proof. After a change of variable and using the continuity of fluid stress, We write $\mathcal{R}_{1}^{(2)}$

$$
\mathcal{R}_{1}^{(2)}=\varepsilon h\left(H_{2}-\left.\tilde{u}_{x}\right|_{z=\varepsilon}\right)+\int_{0}^{\varepsilon} J(\tilde{p}(t, x, \bar{z})-\tilde{p}(t, x, \varepsilon)) d \bar{z} .
$$

It is a straightforward computation to prove that

$$
\begin{aligned}
\left|\partial_{x} \mathcal{R}_{1}^{(2)}\right| \leq & C \varepsilon\left(\left|H_{2}\right|_{1}+\left|u_{x}(\cdot, \varepsilon)\right|_{0}+\left|u_{x x}(\cdot, \varepsilon)\right|_{0}\right) \\
& +C \sqrt{\varepsilon}\left(\|\tilde{p}-\tilde{p}(\cdot, \varepsilon)\|_{0}+\left\|\tilde{p}_{x}-\tilde{p}_{x}(\cdot, \varepsilon)\right\|_{0}\right) .
\end{aligned}
$$

From the Poincaré inequality and estimates on $\|\nabla \tilde{p}\|_{0}$ and $\left\|\nabla \tilde{p}_{x}\right\|_{0}$ together with the estimates on $\mathrm{H}_{2}$, we deduce that

$$
\left|\partial_{x} \mathcal{R}_{1}^{(2)}\right|_{0} \leq C \sqrt{\varepsilon}\left(\sum_{j=0}^{2}\left\|\nabla \partial_{x}^{j} \tilde{u}\right\|_{0}+\left\|\nabla \tilde{u}_{t}\right\|_{0}\right) .
$$


Following the method to obtain an estimate of $\mathcal{R}_{3}$, one shows that $\partial_{x} \mathcal{R}_{1}^{(1)}$ satisfies

$$
\left|\partial_{x} \mathcal{R}_{1}^{(1)}\right|_{0} \leq C \sqrt{\varepsilon}\left(\sum_{j=0}^{2}\left\|\nabla \partial_{x}^{j} \tilde{u}\right\|_{0}+\left\|\nabla \tilde{u}_{t}\right\|_{0}\right) .
$$

Furthermore, one can prove that

$$
\left|\varepsilon \int_{0}^{h} \partial_{x} u_{x}(t, x, \zeta) d \zeta\right|_{0} \leq C \varepsilon^{\frac{3}{2}}\left(\left\|\nabla \tilde{u}_{x}\right\|_{0}+\left\|\nabla \tilde{u}_{x x}\right\|_{0}\right) .
$$

Then, there remains to deal with the capillary term $I_{\bar{\kappa}}=\bar{\kappa}\left(\left(1-\left(1+\varepsilon^{2} h_{x}^{2}\right)\right)^{-\frac{3}{2}} h_{x} h_{x x}\right)$. Here we use the fact that $\left|h_{x x}\right|_{0}$ is a bounded function of time and $\left|h_{x}\right|_{\infty} \leq\left|h_{x x}\right|_{0}$ : one can prove that $\left|I_{\bar{\kappa}}\right| \leq C \bar{\kappa} \varepsilon^{2}\left|h_{x x}\right|_{0}$. From the estimates on $\left|h_{x x}\right|_{0}$, we obtain

$$
\left|I_{\bar{\kappa}}\right| \leq C \varepsilon^{\frac{3}{2}}\left(\sum_{j=0}^{2}\left\|\nabla \partial_{x}^{j} \tilde{u}\right\|_{0}+\left\|\nabla \tilde{u}_{t}\right\|_{0}\right) .
$$

This completes the proof of the lemma 7 .

We finish the proof of the Theorem 2 with the estimate of $\mathcal{R}_{2}$.

Lemma 8. The function $\mathcal{R}_{2}$ satisfies the estimate

$$
\left\|\mathcal{R}_{2}\right\|_{L^{2}\left(0, \infty ; L^{2}(\mathbb{T})\right)} \leq C \sqrt{\varepsilon}
$$

Proof. The function $\mathcal{R}_{2}$ is written $\mathcal{R}_{2}=\partial_{z} \mathcal{R}_{2}^{(1)}(0)+\frac{2}{h^{2}} \int_{0}^{h} \mathcal{R}_{2}^{(1)}(t, ., \zeta) d \zeta$. We first consider the function $\partial_{z} \mathcal{R}_{2}^{(1)}(0)$ defined as

$$
\begin{aligned}
\partial_{z} \mathcal{R}_{2}^{(1)}(0)= & \frac{\varepsilon}{\mathbf{R e}} \int_{0}^{h} u_{x x}-u_{x x}^{(0)}-\int_{0}^{h} \frac{2}{\mathbf{R e}}\left(p-p^{(0)}\right)_{x} \\
& -\frac{\varepsilon}{\mathbf{R e}}\left(\left(w_{x}(h)+\frac{w_{z}(h)}{1-\varepsilon^{2} h_{x}^{2}}\right)-\left(w_{x}^{(0)}(h)+\frac{w_{z}^{(0)}(h)}{1-\varepsilon^{2} h_{x}^{2}}\right)\right) \\
& +\int_{0}^{h}\left(u-u^{(0)}\right)_{t}+\left(u u_{x}-u^{(0)} u_{x}^{(0)}\right)+\left(w u_{z}-w^{(0)} u_{z}^{(0)}\right),
\end{aligned}
$$

First, following the proof of Lemma 7, one can show that

$$
\begin{aligned}
& \left|\frac{\varepsilon}{\mathbf{R e}} \int_{0}^{h} u_{x x}-u_{x x}^{(0)}-\int_{0}^{h} \frac{2}{\mathbf{R e}}\left(p-p^{(0)}\right)_{x}\right|_{0} \leq C \sqrt{\varepsilon}\left(\sum_{j=0}^{2}\left\|\nabla \partial_{x}^{j} \tilde{u}\right\|_{0}+\left\|\nabla \tilde{u}_{t}\right\|_{0}\right) \\
& \left|\int_{0}^{h}\left(u u_{x}-u^{(0)} u_{x}^{(0)}\right)+\left(w u_{z}-w^{(0)} u_{z}^{(0)}\right)\right|_{0} \leq C \sqrt{\varepsilon}\left(\sum_{j=0}^{2}\left\|\nabla \partial_{x}^{j} \tilde{u}\right\|_{0}+\left\|\nabla \tilde{u}_{t}\right\|_{0}\right) .
\end{aligned}
$$

Furthermore, we easily show, after a change of variable, that

$$
\left|\int_{0}^{h}\left(u-u^{(0)}\right)_{t}\right|_{0} \leq \frac{C}{\varepsilon}\left(\left|\int_{0}^{\varepsilon}\right| \tilde{u}||_{0}+\left|\int_{0}^{\varepsilon}\right| \tilde{u}_{t}||_{0}\right) .
$$


Then, using Cauchy Schwarz and Poincaré inequalities, one obtains

$$
\left|\int_{0}^{h}\left(u-u^{(0)}\right)_{t}\right|_{0} \leq C \sqrt{\varepsilon}\left(\|\nabla \tilde{u} \mid\|_{0}+\left\|\nabla \tilde{u}_{t}\right\|_{0}\right) .
$$

The boundary term $-\varepsilon\left(w_{x}(h)-w_{x}^{(0)}(h)\right) / \mathbf{R e}$ is written, using divergence free condition and the no slip condition

$$
-\frac{\varepsilon}{\mathbf{R e}}\left(w_{x}(h)-w_{x}^{(0)}(h)\right)=\frac{\varepsilon}{\mathbf{R e}} \int_{0}^{h}\left(u_{x x}-u_{x x}^{(0)}\right) .
$$

That term has been treated previously. Finally, we consider the boundary term

$$
\frac{\varepsilon}{\mathbf{R e}}\left(1-\varepsilon^{2} h_{x}^{2}\right)^{-1}\left(w_{z}(h)-w_{z}^{(0)}(h)\right)=\frac{\varepsilon}{\mathbf{R e}} \int_{0}^{h}\left(u_{x z}-u_{x z}^{(0)}\right) .
$$

After a change of variable, we obtain

$$
\begin{aligned}
\left|\frac{\varepsilon}{\operatorname{Re}}\left(1-\varepsilon^{2} h_{x}^{2}\right)^{-1}\left(w_{z}(h)-w_{z}^{(0)}(h)\right)\right| & \leq C\left(\left|\int_{0}^{\varepsilon}\right| \tilde{u}_{z}||+\left|\int_{0}^{\varepsilon}\right| \tilde{u}_{x}||+|| \int_{0}^{\varepsilon}\left|\tilde{u}_{x z}\right| \mid\right) \\
& \leq C \sqrt{\varepsilon}\left(\left\|\nabla \tilde{u}_{x}\right\|_{0}+\|\nabla \tilde{u}\|_{0}\right) .
\end{aligned}
$$

Finally, the integral term $\int_{0}^{h} \mathcal{R}_{2}^{(1)} / h^{2}$ is estimated similarly. One can prove that

$$
\left|\frac{1}{h^{2}} \int_{0}^{h} \mathcal{R}_{2}^{(1)}\right|_{0} \leq C \sqrt{\varepsilon}\left(\sum_{j=0}^{2}\left\|\nabla \partial_{x}^{j} \tilde{u}\right\|_{0}+\left\|\nabla \tilde{u}_{t}\right\|_{0}\right)
$$

and Lemma 8 is proved.

As a consequence, from Lemmas $6,7,8$, we deduce that the remainder term $\widetilde{\mathcal{R}}$ in the shallow-water system (98) satisfies

$$
\|\widetilde{\mathcal{R}}\|_{L^{2}\left((0, \infty), L^{2}(\mathbb{T})\right)} \leq C \sqrt{\varepsilon}
$$

This concludes the proof of Theorem (2) and the mathematical justification of the shallow water model (98).

4.2. Lubrication models. As a by product of the derivation of shallow water equations, we shall obtain a hierarchy of model with a single equation on the fluid height $\tilde{h}$ : these are lubrication models. First let us recall that $h, v$ satisfies the mass conservation equation

$$
h_{t}+(h v)_{x}=0
$$

As a first approximation, $h v$ satisfies the expansion

$$
h v=2 s \frac{h^{3}}{3}+\int_{0}^{h}\left(u-u^{(0)}\right) .
$$

Substituting equation (105) into (104), one obtains the Burgers type equation

$$
h_{t}+\left(s \frac{h^{3}}{3}\right)_{x}=-\partial_{x}\left(\int_{0}^{h}\left(u-u^{(0)}\right)\right) .
$$


We then deduce from the analysis of the previous section that

$$
-\partial_{x}\left(\int_{0}^{h}\left(u-u^{(0)}\right)\right)=\mathcal{O}(\sqrt{\varepsilon})
$$

in the $L^{2}\left((0, \infty), L^{2}(\mathbb{R})\right)$-norm and this justifies the fact that the Burgers type equation (106) is a first approximation of shallow water flows. Furthermore, we can obtain more accurate models, using the more precise expansion of $h v$ :

$$
(h v)(t, x)=2 s \frac{h^{3}(t, x)}{3}+\varepsilon \int_{0}^{h(t, x)} \bar{u}^{(1)}(t, x, \zeta) d \zeta+\varepsilon \mathbf{R e} \int_{0}^{h(t, x)} \mathcal{R}_{2}^{(1)}(t, x, \zeta) d \zeta,
$$

where $\bar{u}^{(1)}$ is the function

$$
\bar{u}^{(1)}=-2 p^{(0)}-\boldsymbol{\operatorname { R e }}\left(u_{t}^{(0)}+u^{(0)} u_{x}^{(0)}+w^{(0)} u_{z}^{(0)}\right) .
$$

Then, it is a lengthly but straightforward computation that $h$ satisfies the lubrication equation

$$
\partial_{t} h+\partial_{x}\left(\frac{h^{3}}{3}\left(2 s+\varepsilon\left(\operatorname{Re} \frac{8 s^{2}}{5} h^{3}-2 c\right) h_{x}+2 \varepsilon \bar{\kappa} h_{x x x}\right)\right)=\mathcal{R}_{l u b},
$$

and the function $\mathcal{R}_{l u b}$ satisfies the estimate

$$
\left\|\mathcal{R}_{l u b}\right\|_{L^{2}\left(0, \infty ; L^{2}(\mathbb{R})\right)} \leq C \varepsilon^{\frac{3}{2}} .
$$

This justifies the lubrication approximation for shallow water flows.

$$
\partial_{t} h+\partial_{x}\left(\frac{h^{3}}{3}\left(2 s+\varepsilon\left(\operatorname{Re} \frac{8 s^{2}}{5} h^{3}-2 c\right) h_{x}+2 \varepsilon \bar{\kappa} h_{x x x}\right)\right)=0 .
$$

At this stage, one can derive a viscous Burgers equation: let us write $h$ in the form $h=1+\varepsilon \bar{h}\left(\varepsilon(x-2 s t), \varepsilon^{2} \tau\right)$. This is precisely the diffusive scaling used in [15], to derive a viscous Burgers equation from the full Navier-Stokes system. Then one proves that $\bar{h}$ satisfies, up to zeroth order in $\varepsilon$ the equation

$$
\partial_{t} \bar{h}+\alpha \partial_{x}\left(\bar{h}^{2}\right)=\beta \partial_{x x} \bar{h}, \quad \alpha, \beta>0 .
$$

As a conclusion, we recover a result similar to that of H. UECKER [15] in the particular case of periodic functions in the streamwise variable: here we can also prove that the perturbation $\bar{h}$ decays exponentially fast whereas localized solutions of (110) have a self similar decay.

5. Conclusion. In this paper, we have rigourously justified the derivation of a shallow water system. Such proof has been possible due to the iterative scheme which is described in Subsection 2.2. Mathematical justification of the shallow water derivation described in [8] and for which well posedness has been studied in [6] is an open problem. It corresponds to a Navier slip boundary condition assumption on the bottom instead of a no-slip condition with a particular relation between the Reynolds number and the aspect ratio coefficient. Remark also that our proof has been written from the 2D Navier-Stokes equations to a $1 \mathrm{D}$ shallow water systems but the generalization to the 3D-2D case may be done in the same way with higher derivative estimates. The main hypothesis is a non zero surface tension coefficient. Looking at the vanishing surface tension limit is an interesting open problem that is postponed to a forthcoming work. 


\section{REFERENCES}

[1] J.T. Beale, The Initial Value Problem for the Navier-Stokes Equations with a Free Surface, Comm. Pure Appl. Math., 34 (1981), pp. 359-392.

[2] J.T. Beale, Large-Time Regularity of Viscous Surface Waves, Arch. Rational. Mech. Anal., 84 (1984), pp. 307-352.

[3] A. L. Bertozzi And M. Pugh, The lubrication approximation for thin viscous films: Regularity and long-time behavior of weak solutions, Comm. Pure Applied Math., 49 (February, 1996), pp. $85-123$.

[4] F. Bouchut, M. Westdickenberg. Gravity driven shallow water models for arbitrary topography, Comm. in Math. Sci., 2 (2004), pp. 359-389.

[5] M. Boutounet, L. Chupin, P. Noble, J.-P. Vila. Shallow water flows for arbitrary topograph, Comm. Math. Sciences, 6 (2008), pp. 29-55.

[6] D. Bresch, B. Desjardins, Existence of Global Weak Solutions for a $2 D$ Viscous ShallowWater Equations and Convergence to the Quasi-Geostrophic Model, Comm. Math. Phys., 238 (2003), pp. 211-223.

[7] G.P. Galdi, An Introduction to the Mathematical Theory of the Navier-Stokes Equations: Linearized Steady Problems, Springer Tracts in Natural Philosophy, vol 38, (1994).

[8] J.-F. Gerbeau, B. Perthame, Derivation of Viscous Saint-Venant System for Laminar Shallow-Water: Numerical Validation, Discrete Contin. Dyn. Syst. Ser. B, (2001), pp. 89102.

[9] L. Giacomelli, F. Отто. Rigorous lubrication approximation, Interfaces and Free Boundaries, 5 (2003), pp. 483-529.

[10] H. Iто, Best Constants in Körn-Poincaré's Inequalities on a Slab, Math. Methods in the Applied Sciences, 17 (1994), pp. 525-549.

[11] F. MARChe. Derivation of a new two-dimensional shallow water model with varying topography, bottom friction and capillary effects, Eur. J. Mech. B. Fluids, 26 (2007), pp. 49-63.

[12] T. Nishida, Y. Teramoto, H.A. Win, Navier-Stokes flows down an inclined plane: downward periodic motion, J. Math. Kyoto Univ., 33-3 (1993), pp. 787-801.

[13] R. Temam, M. Ziane, Navier-Stokes equations in three-dimensional thin domains with various boundary conditions, Adv. in Diff. Equation, 1 (1996), pp. 499-546.

[14] Y. Teramoto, On the Navier-Stokes Flows Down an Inclined Plane, J. Math. Kyoto Univ., 32-3 (1992), pp. 593-619.

[15] H. Uecker, Self-Similar Decay of Spatially Localized Perturbations of the Nusslet Solutions for the Inclined Film Problem, Arch. Rational Mech. Anal., 184 (2007), pp. 401-447.

[16] J.-P. VILA, Two Moments Closure Equations of Shallow-Water Type for Thin Film Laminar Flow Gravity Driven, in preparation (2007). 
